\title{
Development of a Fixed Abrasive Slicing Technique (FAST) for Reducing the Cost of Photovoltaic Wafers
}

\section{Final Subcontract Report 9 January 1991 - 14 April 1991}

F. Schmid

Crystal Systems, Inc.

Salem, Massachussetts

NREL technical monitor: R. Mitchell

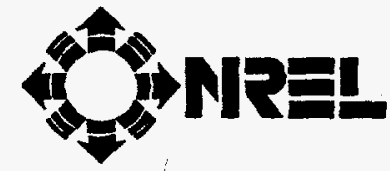

National Renewable Energy Laboratory (formerly the Solar Energy Research Institute) 1617 Cole Boulevard Golden, Colorado 80401-3393

A Division of Midwest Research Institute Operated for the U.S. Department of Energy under Contract No. DE-AC02-83CH10093

Prepared under Subcontract No. XC-1-10057-17

December 1991 
On September 16, 1991, the Solar Energy Research Institute was designated a national laboratory, and its name was changed to the Natlonal Renewable Energy Laboratory.

\title{
NOTICE
}

This report was prepared as an account of work sponsored by an agency of the United States government. Neither the United States government nor any agency thereof, nor any of their employees, makes any warranty, express or implied, or assumes any legal liability or responsibility for the accuracy, completeness, or usefulness of any information, apparatus, product, or process disclosed, or represents that its use would not infringe privately owned rights. Reference herein to any specific commercial product, process, or service by trade name, trademark, manufacturer, or otherwise does not necessarily constitute or imply its endorsement, recommendation, or favoring by the United States government or any agency thereof. The views and opinions of authors expressed herein do not necessarily state or reflect those of the United States government or any agency thereof.

\author{
Printed in the United States of America \\ Available from: \\ National Technical Information Service \\ U.S. Department of Commerce \\ 5285 Port Royal Road \\ Springfield, VA 22161
}

Price: Microfiche A01

Printed Copy A04

Codes are used for pricing all publications. The code is determined by the number of pages in the publication. Information pertaining to the pricing codes can be found in the current issue of the following publications which are generally available in most libraries: Energy Research Abstracts (ERA); Government Reports Announcements and Index (GRA and I); Scientific and Technical Abstract Reports (STAR); and publication NTIS-PR-360 available from NTIS at the above address. 


\section{DISCLAIMER}

Portions of this document may be illegible electronic image products. Images are produced from the best available original document. 
TABLE OF CONTENTS

Page

1.0 Introduction ..................... 1

2.0 Background . . . . . . . . . . . . . . . . . 4

2.1 FAST Slicing . . . . . . . . . . . . . . . . 4

2.2 Expendable Materials Costs for FAST Slicing . . . . 10

3.0 Experimental Results . . . . . . . . . . . . . . 14

3.1 Slicing Tests . . . . . . . . . . . . . . 14

3.2 Analysis of Data . . . . . . . . . . . . . . 28

3.3 Dry Cutting . . . . . . . . . . . . . . . . . . 37

3.4 Correlation of Experimental Data with . . . . . . . 38 Theoretical Analysis

3.4.1 Diamond Size Distribution.......... 38

3.4.2 Penetration of Diamonds Into the Workpiece . . 42

3.5 Degradation Mechanisms of Bladepacks . . . . . . . 45

4.0 Cost Analysis . . . . . . . . . . . . . . . . . . 50

5.0 Results and Discussions . . . . . . . . . . . . 53

6.0 References . . . . . . . . . . . . . . . . . 57 
LIST OF TABLES

Table

Page

I Comparison of the Double-Headed Prototype FAST Slicer . . . . 9 with the Laboratory Slicer in 1981

II Details of Experiments Carried Out on Blade Development . . . 16

III Details of Bladepacks Used in Slicing Experiments . . . . . 19

IV Material utilization, throughput and projected costs . . . . 51 in production for FAST slicing. In this analysis

the bladepack life is assumed to be 5 cuts/bladepack.

$V$ Projected costs of FAST slicing, \$/wafer, showing . . . . . . 52 sensitivity of bladepack life and material utilization on silicon ingot, slicing and total wafer costs for $100 \mathrm{~mm} \times 100 \mathrm{~mm}$ wafer production. Silicon bar costs are assumed to be $\$ 70 / \mathrm{kg}$ and other parameters are the same as Table IV. 
1 Schematic of FAST Slicer . . . . . . . . . . . . . 5

2 Double-Headed Prototype FAST Slicer . . . . . . . . . . . . 8

3 Schematic of electroforming tooling at spacing of 40 . . . . 15 wires per inch with a 0.008 inch core wire placed in $60^{\circ} \mathrm{V}$-grooves.

4 Cutting rate as a function of time for $45 \mu \mathrm{m} . . . . \quad . .$. diamond-plated wirepack used in Experiment 1.

5 Cutting effectiveness of mating bladepack used in . . . . . Experiment 1 showing cutting rate with elapsed time during Experiment 2.

6 Cutting effectiveness for a bladepack using tungsten . . . . core wire in slicing two silicon blocks in Experiments 3 and 4.

8 Examination of diamond-plated wire showing high . . . . . . . concentration of diamonds.

9 Examination of a diamond-plated wire section tilted about. $20^{\circ}$ to observe the elevation of diamonds.

10 A low magnification examination of a diamond-plated . . . . 33 wire showing high diamond concentration.

11 SEM examination of five consecutive sections of . . . . . . . 34 silicon block showing scratches after abrasion with diamond-plated plate.

12 A magnified view of a $45 \mu \mathrm{m}$ diamond showing faceted faces . . 36

13 RBEI examination of a diamond-plated plate after . . . . . . 36 vigorous rubbing showing that only a few diamonds were contacting the workpiece.

14 A typical distribution of $45 \mu \mathrm{m}$ diamonds . . . . . . . . . . 39

15 Two views of a wire after crushing between flat rollers. . . 44 showing similar protrusion of diamonds. 
Figure

Page

16 SEM examination of a wire after use showing that there . . . 47 is no diamond pull-out.

17 Optical examination of a CBN coated wire after use . . . 48 showing wear flats. 


\subsection{Introduction}

The photovoltaic industry has grown rapidly during the last decade. Photovoltaics is now an important alternate energy source, and modules are in use in commercial installations. Several countries are planning on photovoltaics as a future energy source. Examples are Italy and developing countries such as India and Brazil which have ambitious plans to use photovoltaics. In the applications area consumer products such as watches and calculators are becoming dwarfed by remote applications like microwave repeater stations and lighthouses and applications such as powering vacation homes, water buoys, water pumping and street lighting. The photovoltaic industry has been growing and this industry's growth rate is projected at 20-30 percent annually. It is now recognized that to broaden photovoltaic (PV) applications, it is necessary to lower the cost of modules without sacrificing high performance.

Since the mid 1970's when solar cells were fabricated using high-purity single-crystal silicon wafers, a number of alternate technologies have been developed. During the 1980's the material options for solar cells were crystalline silicon, amorphous silicon and other materials utilized as thin films. It is now recognized that the alternate materials have to undergo development, testing and a learning curve prior to setting up major production facilities. Amorphous silicon is limited by lower efficiency than crystalline silicon and it degrades with time. The best choice for the near term is therefore crystalline silicon and the photovoltaic industry recognizes that only crystalline silicon can meet the requirements of photovoltaic modules for near future applications. To lower cost the high power density must be combined with efficient high throughput in production. The photovoltaic industry is currently relying on the use of silicon wafers produced by the Czochralski (Cz), multicrystalline growth or multicrystalline techniques. An essential component 
of all of these techniques is that the silicon is available in the form of an ingot and has to be sliced into wafers for production of solar cells and modules. Other techniques Involve some sort of ribbon growth where sheet silicon is produced directly from the melt, but these are limited by low throughput and/or low efficiency and are therefore still in the development stage or have been dropped. The ribbon techniques do not involve a slicing step, but none of these techniques are being practiced in production. The heart of photovoltaics is therefore the silicon wafers sliced from single or multicrystalline ingots. The silicon wafers represent over 50 percent of the module cost and slicing represents nearly half of the wafer cost. Reduction of the silicon wafer cost is critical for lowering the cost of energy production by PV.

Ingot growth technologies for crystalline silicon have shown considerable progress during the last decade to produce square high efficiency ingots at low cost. To realize this progress the ingots must be efficiently sliced into wafers. Slicing techniques utilized in production today have large kerf loss and/or high consumable costs to the point that the slicing cost is more than half the wafer cost. Therefore, slicing of crystalline silicon ingots is still considered to be an obstacle to achieving further cost reduction of photovoltaic modules. It is desirable to develop an effective slicing technique to slice thin wafers with low kerf at low cost. If these kerf and wafer objectives are achieved, the contribution of silicon meltstock and ingot growth costs to the wafer costs are lessened because less silicon material is required. Today a substantial portion of the total cost of the wafer is in the wafering.

Among the slicing techniques available to the industry, only three approaches are currently being pursued. The industry standard, internal diameter (ID), has been optimized, and significant further cost reductions are not anticipated. Improvements have been made in Multi-Wire Slurry (MWS) technique; 
however, the expendable materials cost (wire, SiC slurry and oil) control the cost of slicing. Moreover, the disposal of silicon, silicon carbide and oil mixture presents environmental problems.

A third approach, the Fixed Abrasive Slicing Technique (FAST), is currently at a prototype production stage. In FAST the expendable materials costs are reduced by using a diamond-plated wirepack, while the advantages of MWS are retained.

In the FAST approach a multi-wire bladepack is reciprocated across the silicon workpiece, and slicing is achieved by using fixed diamond on the wires as an abrasive and water as a coolant. In the bladepack equal spacing and tension of the wires is achieved and the diamonds are plated onto the wires in the cutting edge only. The diamonds on the wire protect it from abrasion and failure during slicing due to wear, and the wires do not fail due to fatigue since they do not wind around rollers. With this approach the kerf is reduced and the amount of diamonds used is decreased. It is recognized in the industry that diamond is very effective as an abrasive for slicing silicon.

Major developments in the area of fabrication of wirepacks, design of FAST slicer with high throughput, and slicing of crystalline silicon blocks has demonstrated that FAST is an effective technique for slicing crystalline wafers for photovoltaic applications. It is, however, necessary to develop FAST so that it can be utilized in production by the industry. One of the areas for further development is the use of multi-wire bladepacks for slicing multiple blocks of crystalline silicon so that the consumable bladepack cost and kerf loss can be reduced. 


\subsection{Background}

\subsection{FAST Slicing}

The FAST approach involves holding a diamond-coated multi-wire bladepack in a frame and reciprocating the frame across a workpiece. Initially this approach was utilized for slicing sapphire crystals. The adaptation of FAST for slicing silicon was established under a DOE/JPL program. Initially a multiblade slurry slicer was modified to demonstrate FAST slicing. It was observed that the massive bladehead required for tensioning of blades limited high reciprocation speeds necessary for FAST slicing and a laboratory FAST slicer was designed and fabricated. Some of the key elements of this slicer were a lightweight bladehead to achieve high reciprocation speeds, rocking of the crystal to minimize contact length between the wire and the crystal, straight line motion of the bladehead to minimize vibration for accuracy of slicing, and a sensitive feed mechanism for slicing with low feed forces. A schematic of the FAST slicer is shown in Figure 1.

A key element of FAST slicing is its wirepack with equal spacing and tension. A simple process for forming a wirepack ${ }^{2}$ was developed in which the cumulative errors in spacing of wires was minimized. Two approaches of fixing diamonds onto the wire were developed. The first approach involved plating of diamonds in a nickel matrix and the second approach was impregnation of diamonds in a soft sheath covering the core wire. The impregnation approach showed that the diamonds were easily pulled out during slicing. Plating of diamonds after impregnation only delayed the diamond pul1-out problem and this approach was dropped in favor of the plating approach.

Initially the entire circumference of the wire was plated with diamonds. With this approach the rollers on either side of the workpiece used to guide the wires were abraded. The accuracy of slicing also reduced as slicing of the 


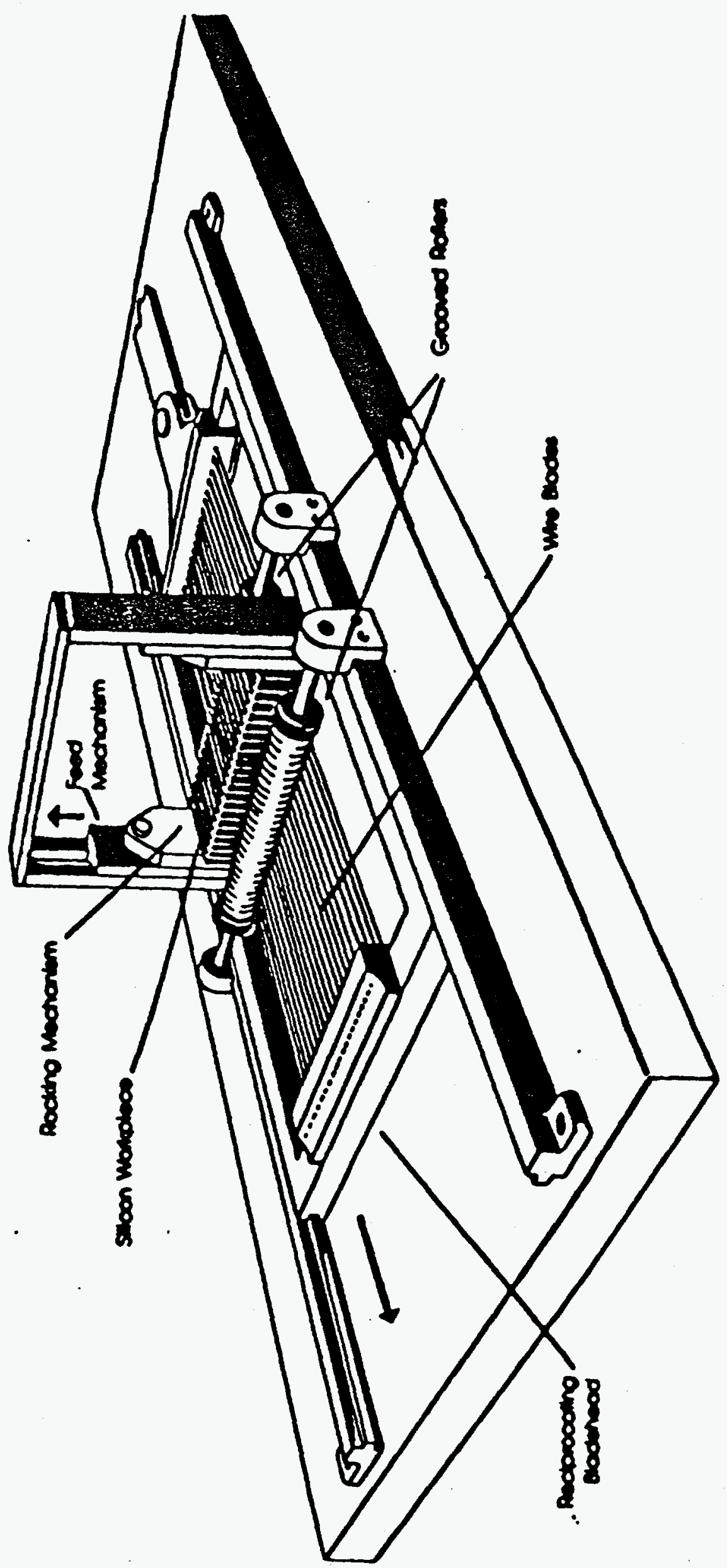

¿े 
workpiece progressed. An electroforming technique ${ }^{3}$ was developed in which the diamonds were plated only on the cutting edge of the wires. Under these conditions the portion of the wire contacting the guide rollers was not coated with diamonds. The electroforming technique minimized wear of the grooved guide rollers, aided seating of the wires in the grooved rollers and improved accuracy of slicing. Further work with the electroforming technique showed that the shape of the plating on the wires could be controlled so that the kerf could be minimized even when larger diamonds were utilized. This approach also allowed use of multiple layers of diamonds without increasing the kerf.

The key element of FAST slicing is to maximize the pressure at the diamond tip during slicing. It was found that the cutting effectiveness degraded rapidly as the contact length between the wires and the workpiece increased. The concept of rocking the workpiece 4.5 was therefore introduced. With this approach a radial cut profile is formed during FAST slicing when the crystal is rocked. The contact length is reduced even as the size of the crystal being sliced is increased. Reduction of the contact length maximizes the pressure at the diamond tip so that effective slicing can be carried out even for large crystals.

A correlation between the feed forces on the crystal, deflection of the wires, contact length and rocking angle was developed ${ }^{6}$. This data showed that larger rocking angles are required to slice larger workpiece size. This model was further developed in that the rocking speed has to be increased to improve cutting effectiveness of the wires.

Characterization of FAST sliced silicon wafers showed that the surface damage $^{7}$ in the wafers was limited to 3 to $5 \mu \mathrm{m}$. This is the lowest surface damage observed for any slicing technique. It has also been shown that FAST can be used for effective slicing of GaAs, Ge, CdTe and other expensive materials. Large silicon crystals were sliced by FAST to produce thin wafers with minimum 
kerf. It was shown that 64 wafers/inch $(25 / \mathrm{cm})$ could be sliced ${ }^{8}$ with a $10 \mathrm{~cm}$ diameter workpiece and that the kerf could be reduced to less than $150 \mu$. In smaller sizes it was shown that $100 \mu$ thick wafers could be sliced ${ }^{9}$ with FAST.

Once FAST slicing was demonstrated in the laboratory and it was shown that high quality thin wafers could be produced with low kerf, a major development was undertaken in 1989 as a cooperative effort with BP Solar Group. The emphasis in this development program was to design and fabricate a prototype FAST slicer with increased throughput and potentially lower cost in production. This slicer (Figure 2) consists of three modules. A Cardan gear system drives two slicing head modules. In this configuration the two slicing modules balance the forces and two silicon bars can be sliced simultaneously. The prototype slicer has the capacity to slice 30 inches of silicon bar per slicing run. Effective slicing with this slicer has been demonstrated at 40 wafers/inch $(16 / \mathrm{cm})$ of silicon so that 1,200 wafers can be produced per run. The slicer is designed to slice 10 $\mathrm{cm}$ square and $12.5 \mathrm{~cm}$ square cross-section silicon blocks. Thinner wafers can be sliced by changing the spacing of the wires in the bladepack.

The features of the prototype slicer are compared to the laboratory slicer in Table I. During slicing with the prototype slicer, it was observed that the cutting effectiveness of the bladepack degraded as slicing progressed. An $R \& D$ program was initiated with the laboratory slicer to develop bladepacks to slice multiple silicon bars. Initially emphasis was placed on the plating of diamonds to optimize the bond between the diamond and nickel matrix so that diamonds are not pulled out during slicing. Improvements were also made in cleaning the core wire prior to plating so that a tenacious bond was formed between the core wire and the nickel matrix to prevent stripping plating from the core wire. Variables such as core wire material, size of core wire, size of diamonds, type of diamonds, thickness of nickel plating, hardness of plating, plating parameters, 


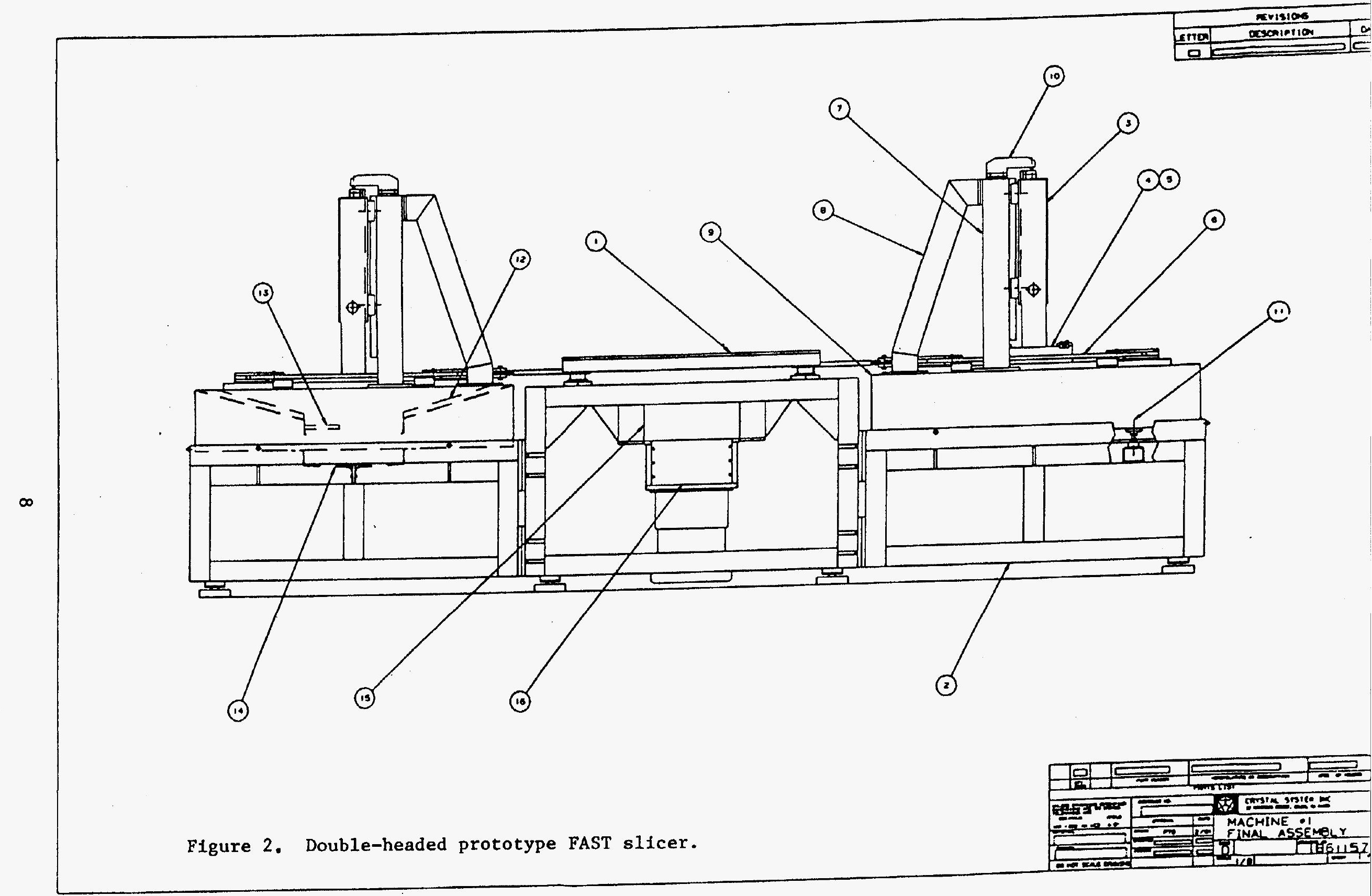


Table I. Comparison of the double-headed prototype FAST slicer with the laboratory slicer in 1981.

\section{FAST}

\# of cutting heads

Bar length per run, cm

\# of wafers/cm capability

\# of wafers/run capability

Bar size capability, $\mathrm{mm}$

\# of $100 \mathrm{~mm} \times 100 \mathrm{~mm}$ wafers/hr.

\section{Present slicing:}

\# of wafers/run

Wafer size, mm

\section{Sl1cer}

1

10

25

250

$100 \times 100$

15
Prototype S11cer

2

76

25

1,920

$125 \times 125$

115

1,200

$100 \times 100$ 
diamond concentration and slicing parameters were optimized to maintain cutting effectiveness of the diamond-plated wirepacks. Experiments were carried out by slicing approximately $1 \mathrm{~cm}$ wide silicon blocks. This size corresponds to the contact length during slicing; therefore, it was not necessary to rock the workpiece for these experiments. It was observed that the initial cutting effectiveness of the diamonds was good; however, as slicing progressed, the cutting effectiveness was reduced. The degradation of bladepacks with friable diamonds was not as severe as with synthetic or natural diamonds. From experiments it was observed that after the bladepack had lost cutting effectiveness, it could be revived by dressing.

The development of the high throughput double-headed FAST slicer has shown that slicing costs can be reduced. Slicing thin wafers with low kerf improves material utilization, thereby reducing the cost of slicing and the cost factor contribution of silicon meltstock and ingot growth towards the total cost of silicon wafers. At present the expendable materials costs are still high, and it is necessary to reduce these costs so that an economic FAST slicer can be used in production. If techniques can be developed so that the wirepack can be utilized to slice multiple silicon blocks, then the expendable materials costs will be reduced significantly.

\subsection{Expendable Materials Costs for FAST Slicing}

During the development of FAST technology, it was evident that the contribution of equipment and labor costs to the total cost of the wafer would be considerably lower than other slicing techniques. It was shown that the cost of wafers could be reduced using FAST as the material utilization was high and surface damage in the wafers was low. As the development of FAST technology progressed, it appeared that with a double-headed FAST slicer, high throughput could be achieved. 
Prior to the start of the present program, all of the essential cost elements discussed above were achieved and demonstrated in prototype production. The only remaining element of the cost of wafers is the expendable materials cost. In FAST slicing the major expendable materials costs are for diamondplated wirepacks. By electroforming techniques the amount of diamond used for diamond plating has been reduced. However, it is essential that the wirepack slice through multiple silicon blocks to reduce the expendable materials costs per slice and thereby make FAST the most cost effective technology for largescale production of silicon wafers for solar cell applications.

It is known that fixed diamond abrasive stands up during slicing of silicon. This is demonstrated by the ID technology where up to 4,000 wafers of 4-inch diameter are routinely sliced in production using a single layer of electroplated diamonds on a blade. It is recognized that very high feed forces and high rotational velocity of the blade in ID slicing differs from the FAST approach. If the wirepack can be used for effectively slicing multiple silicon blocks, the contribution of expendable materials costs (i.e., bladepack) to the wafer costs will be reduced significantly. The economics will be favorable for FAST slicing if five $10 \mathrm{~cm} \times 10 \mathrm{~cm}$ silicon blocks can be sliced. However, if ten such blocks can be sliced, the cost of FAST slicing will be considerably lower than other slicing techniques. An important consideration for FAST slicing is that water is used as a coolant; therefore, the by-product is a mixture of silicon swarf and water which can be disposed of without concern for environmental problems, unlike MWS, where the by-product is a mixture of oil, silicon carbide and silicon.

Once it was recognized that diamonds can be fixed on wires by electroplating, the main degradation mechanism of wire blades was diamond pullout ${ }^{10}$; therefore, emphasis was placed on the plating process so that the 
nickel plating was developed to hold diamonds during FAST slicing. Improvement was observed so that three silicon blocks of $10 \mathrm{~cm}$ diameter could be sliced using the same wirepack ${ }^{11}$. It was observed that the slicing effectiveness for the second and third blocks was reduced as the average slicing rate was less than $100 \mu \mathrm{m}$ per minute and the yield of wafers was reduced. At that stage major improvements were made to the FAST slicer so that the crystal was rocked over a wider angle, a more sensitive feed mechanism was installed, and a completely new drive system was incorporated. Improvements in the slicer allowed effective slicing of $10 \mathrm{~cm} \times 10 \mathrm{~cm}$ silicon blocks. Further improvements in the plating technology showed that diamond pullout was no longer the failure mechanism for the wirepacks even after it had been used for slicing multiple silicon blocks; however, it was observed that the slicing rate decreased with time and the yield of wafers decreased with subsequent blocks.

A program on wire blade development was initiated to understand the degradation mechanism of the wirepacks. In this program $1 \mathrm{~cm}$ wide silicon bars were sliced without rocking. The results of this program are summarized below.

- Very high initial slicing rates (over $500 \mu \mathrm{m}$ per minute) were observed; however, the slicing rate decreased with time.

- For feed forces of 40 to 80 grams per wire, higher slicing rates were observed for higher feed forces.

- The slicing effectiveness of $60 \mu \mathrm{m}$ diamonds was better than $45 \mu \mathrm{m}$ diamonds.

- For the same size diamonds the slicing effectiveness of friable-type diamonds was better than natural or synthetic diamonds.

- Diamond concentration on the wire did not show significant difference in slicing effectiveness. 
- After a wirepack had shown deterioration in slicing effectiveness, it could be revived by dressing.

This data formed the basis for understanding the mechanism for degradation of slicing effectiveness of the wirepack during slicing. 


\subsection{Experimental Results}

Based upon the background results discussed above, slicing of $10 \mathrm{~cm}$ square cross-section silicon blocks was carried out during the present program. The effort involved slicing tests, analysis of data, correlation of experimental data with theoretical analysis, development of a model for increasing the life of bladepacks, cost analysis on the impact of bladepack life to the slicing costs, and development of a solution to the problem of reducing expendable materials costs by increasing the life of the bladepacks.

\subsection{Slicing Tests}

During the program a number of slicing tests were carried out to understand the mechanism which limits the life of the bladepacks during FAST s1icing. The parameters that were tested were diamond size, diamond type, diamond concentration, plating thickness and substrate material. A11 bladepacks except \#22 were made with electroforming tooling with a spacing of 40 wires per inch and a 60 degree angle for plating as shown in Figure 3.

The laboratory FAST slicer was utilized for the slicing tests so that data could be obtained by slicing approximately $100 \mathrm{~mm}$ long sections of $100 \mathrm{~mm} \times 100$ mm silicon blocks. Emphasis was placed on correlating this data with the bladepacks produced for the high-throughput FAST slicer. For example, tooling for plating of 15-inch wide bladepacks was appropriate for plating two matching 4-inch wide bladepacks for the laboratory slicer at the same time. The results of the slicing tests carried out are shown in Table II, and the data on the bladepacks is shown in Table III.

In the slicing tests carried out, low feed force was used initially to prevent breakage and achieve desired curvature of the cut profile starting from straight top surface of the $10 \mathrm{~cm}$ square bars. The feed force was increased to approximately 65 grams per wire to achieve high cutting rates. With this 


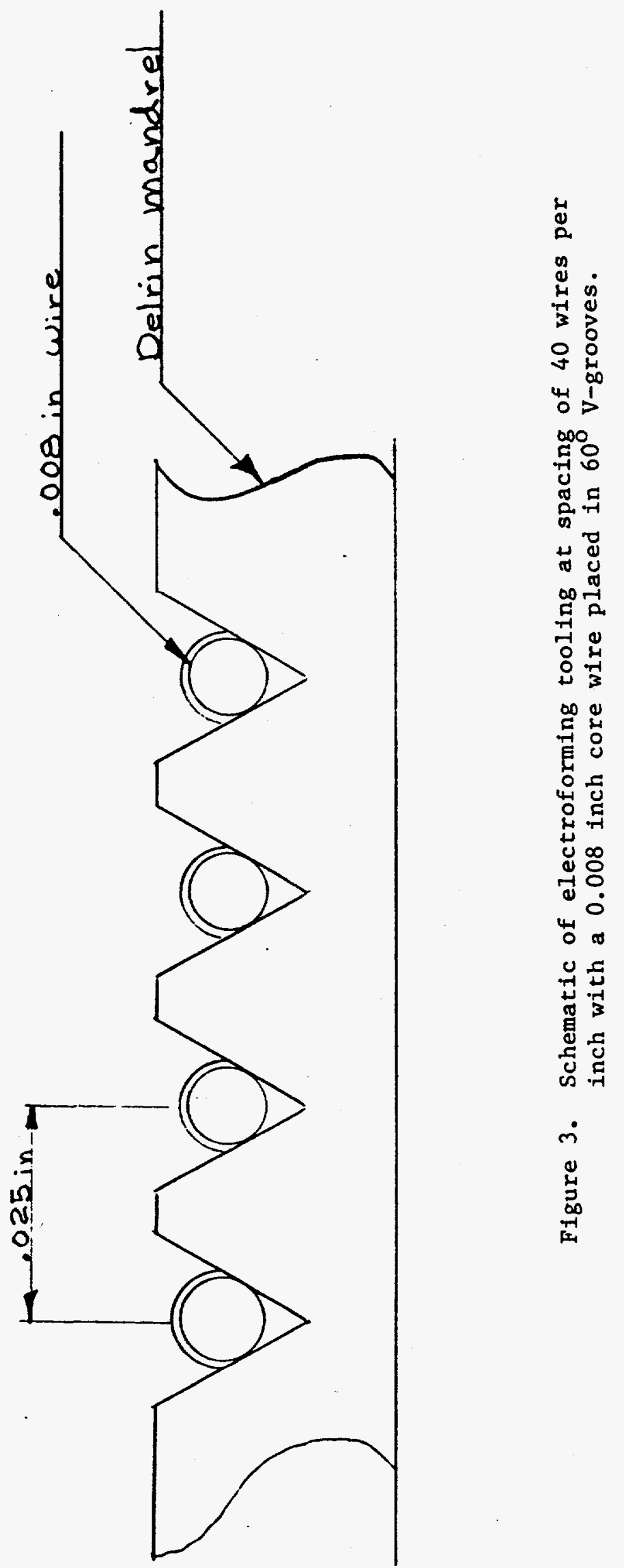


Table II. Details of Experiments Carried out on Blade Development.

\begin{tabular}{|c|c|c|c|c|c|c|c|c|c|}
\hline$\underset{\#}{\text { Expt. }}$ & $\begin{array}{l}\text { Blade- } \\
\text { pack } \\
\text { ID } \#\end{array}$ & $\begin{array}{l}\text { Utting Rate } \\
\text { Beginning } \\
\text { (mils/min) }\end{array}$ & $\begin{array}{l}\text { at Full Load } \\
\text { Ending } \\
\text { (mils/min) }\end{array}$ & $\begin{array}{l}\text { Max } \\
\text { Load } \\
\text { (g/ } \\
\text { wire) }\end{array}$ & $\begin{array}{l}\text { Avg. Cutting } \\
\text { Rate \& Total } \\
\text { Time of cut } \\
\text { (mils/min) } \\
\text { (hrs:min) }\end{array}$ & $\begin{array}{l}\text { Wafers } \\
\text { Lost } \\
\text { During } \\
\text { Run }\end{array}$ & $\begin{array}{l}\text { Wafers } \\
\text { Lost } \\
\text { at } \\
\text { Dismount }\end{array}$ & $\begin{array}{l}\text { \% Yield } \\
\text { Whole } \\
\text { Wafer }\end{array}$ & Remarks \\
\hline 1 & $\begin{array}{l}214 A- \\
071090\end{array}$ & 5.666 & 4.847 & $60 \mathrm{~g} / \mathrm{w}$ & $4.832 / 13: 55$ & 10 & 12 & $86 \%$ & $45 \mu \mathrm{m}$ \\
\hline 2 & $\begin{array}{l}214 \mathrm{~B}- \\
071090\end{array}$ & 4.035 & 4.039 & $60 \mathrm{~g} / \mathrm{w}$ & $3.576 / 19: 25$ & 11 & 15 & $84 \%$ & $\begin{array}{l}\text { Mating } \\
\text { Bladepack }\end{array}$ \\
\hline 3 & $\begin{array}{l}215 A- \\
072590\end{array}$ & 4.048 & 4.055 & $60 \mathrm{~g} / \mathrm{w}$ & $4.864 / 14: 19$ & 11 & 5 & $89 \%$ & $\begin{array}{l}\text { tungsten } \\
\text { wire }\end{array}$ \\
\hline 4 & $\begin{array}{l}215 A- \\
072590-2\end{array}$ & 4.033 & 4.857 & $60 \mathrm{~g} / \mathrm{w}$ & $3.255 / 20: 46$ & 15 & $?$ & $-90 \%$ & 2nd Use \\
\hline 5 & $\begin{array}{l}215 B- \\
072590\end{array}$ & 4.825 & 4.087 & $60 \mathrm{~g} / \mathrm{w}$ & $4.179 / 17: 32$ & 15 & 7 & $86 \%$ & $\begin{array}{l}\text { Mating } \\
\text { Bladepack }\end{array}$ \\
\hline 6 & $\begin{array}{l}221 B- \\
091390\end{array}$ & 4.440 & 4.861 & $60 \mathrm{~g} / \mathrm{w}$ & $3.823 / 17: 34$ & 8 & 15 & $86 \%$ & \\
\hline 7 & $\begin{array}{l}228 \mathrm{~A}- \\
103190\end{array}$ & 5.652 & 6.068 & $67 \mathrm{~g} / \mathrm{w}$ & $1.773 / 3: 18$ & Mass & Destruct & $0 \%$ & \\
\hline 8 & $\begin{array}{l}228 A- \\
103190-2\end{array}$ & 4.485 & 4.052 & $67 \mathrm{~g} / \mathrm{w}$ & $2.926 / 22: 42$ & $?$ & 40 & $-75 \%$ & 2nd Use \\
\hline 9 & $\begin{array}{l}227 A- \\
103090\end{array}$ & 5.272 & 4.038 & $67 \mathrm{~g} / \mathrm{w}$ & $3.332 / 19: 40$ & 6 & 6 & $92.5 \%$ & $\begin{array}{l}\text { natural dia. } \\
\text { moderate } \\
\text { friable }\end{array}$ \\
\hline 10 & $\begin{array}{l}227 A- \\
103090-2\end{array}$ & 4.461 & 2.042 & $67 \mathrm{~g} / \mathrm{w}$ & $2.024 / 13: 06$ & Run 7 & Aborted & $0 \%$ & 2nd Use \\
\hline 11 & $\begin{array}{l}227 A- \\
103090-3\end{array}$ & 3.239 & 2.837 & $67 \mathrm{~g} / \mathrm{w}$ & $\begin{array}{l}2.533 / 25: 23 \\
16\end{array}$ & 14 & 27 & $71 \%$ & $\begin{array}{l}\text { 3rd Use } \\
\text { After Dressing }\end{array}$ \\
\hline
\end{tabular}




\begin{tabular}{|c|c|c|c|c|c|c|c|c|c|}
\hline$\underset{\#}{\operatorname{Expt}}$ & $\begin{array}{l}\text { Blade- } \\
\text { pack } \\
\text { ID \# }\end{array}$ & $\begin{array}{l}\text { utting Rate } \\
\text { Beginning } \\
\text { (mils/min) }\end{array}$ & $\begin{array}{l}\text { at Full Load } \\
\text { Ending } \\
\text { (mils/min) }\end{array}$ & $\begin{array}{l}\text { Max } \\
\text { Load } \\
\text { (g/ } \\
\text { wire) }\end{array}$ & $\begin{array}{l}\text { Avg. Cutting } \\
\text { Rate \& Total } \\
\text { Time of Cut } \\
\text { (mils/min) } \\
\text { (hrs:min) }\end{array}$ & $\begin{array}{l}\text { Wafers } \\
\text { Lost } \\
\text { During } \\
\text { Run }\end{array}$ & $\begin{array}{l}\text { Wafers } \\
\text { Lost } \\
\text { at } \\
\text { Dismount }\end{array}$ & $\begin{array}{l}\text { \% Yield } \\
\text { Whole } \\
\text { Wafer }\end{array}$ & Remarks \\
\hline 12 & $\begin{array}{l}226 \mathrm{~B}- \\
102690\end{array}$ & 4.894 & 5.271 & $67 \mathrm{~g} / \mathrm{w}$ & $3.784 / 17: 31$ & 2 & 2 & $96 \%$ & $\begin{array}{l}\text { friable } \\
\text { diamond }\end{array}$ \\
\hline 13 & $\begin{array}{l}226 \mathrm{~B}- \\
102690-2\end{array}$ & 5.288 & 3.649 & $67 \mathrm{~g} / \mathrm{w}$ & $2.854 / 23: 17$ & 9 & 11 & $87 \%$ & 2nd Use \\
\hline 14 & $\begin{array}{l}241 A- \\
11291\end{array}$ & 6.899 & 4.867 & $67 \mathrm{~g} / \mathrm{w}$ & $4.588 / 14: 38$ & 5 & 17 & $86 \%$ & \\
\hline 15 & $\begin{array}{l}241 A- \\
11291-2\end{array}$ & 2.437 & 2.444 & $47.5 \mathrm{~g} / \mathrm{w}$ & $1.715 / 3: 57$ & Run & Aborted & $0 \%$ & 2nd Use \\
\hline 16 & $\begin{array}{l}241 B- \\
11291\end{array}$ & 4.056 & 3.649 & $60 \mathrm{~g} / \mathrm{w}$ & $3.914 / 17: 04$ & 4 & 20 & $85 \%$ & $\begin{array}{l}\text { Mating } \\
\text { Bladepack } \\
\text { to Expt. } 15\end{array}$ \\
\hline 17 & $\begin{array}{l}241 \mathrm{~B}- \\
11291-2\end{array}$ & 2.441 & 2.033 & $60 \mathrm{~g} / \mathrm{w}$ & $2.832 / 23: 25$ & 6 & $\sim 59$ & $58 \%$ & 2nd Use \\
\hline 18 & $\begin{array}{l}242 \mathrm{~B}- \\
112291\end{array}$ & 4.475 & 2.431 & $60 \mathrm{~g} / \mathrm{w}$ & $2.555 / 25: 37$ & 10 & 22 & $80 \%$ & $\begin{array}{l}30 \mu \mathrm{m} \\
\text { natural }\end{array}$ \\
\hline 19 & $\begin{array}{l}247 A- \\
022591\end{array}$ & 4.842 & 2.437 & $60 \mathrm{~g} / \mathrm{w}$ & $1.590 / 34: 10$ & & & $0 \%$ & $\begin{array}{l}\text { DuPont dia. } \\
\text { most friable }\end{array}$ \\
\hline 20 & $\begin{array}{l}248 A- \\
022691\end{array}$ & 4.883 & 4.052 & $60 \mathrm{~g} / \mathrm{w}$ & $3.729 / 17: 54$ & 5 & 10 & $90 \%$ & \\
\hline 21 & $\begin{array}{l}248 A- \\
022691-2\end{array}$ & 3.261 & 4.051 & $66 \mathrm{~g} / \mathrm{w}$ & $2.965 / 22: 30$ & 16 & 44 & $62 \%$ & 2nd Use \\
\hline 22 & $\begin{array}{l}249 A- \\
030791\end{array}$ & 4.071 & 4.459 & $50.9 \mathrm{~g} / \mathrm{w}$ & $\begin{array}{c}3.788 / 17: 37 \\
17\end{array}$ & 52 & 26 & $60 \%$ & \\
\hline
\end{tabular}


Table II continued.

\begin{tabular}{|c|c|c|c|c|c|c|c|c|c|}
\hline$\underset{\#}{\operatorname{Exp} t .}$ & $\begin{array}{l}\text { 3lade- } \\
\text { pack } \\
\text { ID \# }\end{array}$ & $\begin{array}{c}\text { Cutting Rate } \\
\text { Beginning } \\
\text { (mils/min) }\end{array}$ & $\begin{array}{l}\text { at Full Load } \\
\text { Ending } \\
\text { (mils/min) }\end{array}$ & $\begin{array}{l}\text { Max } \\
\text { Load } \\
\text { (g/ } \\
\text { wire) }\end{array}$ & $\begin{array}{l}\text { Avg. Cutting } \\
\text { Rate \& Total } \\
\text { Time of Cut } \\
\text { (mils/min) } \\
\text { (hrs:min) }\end{array}$ & $\begin{array}{l}\text { Wafers } \\
\text { Lost } \\
\text { During } \\
\text { Run }\end{array}$ & $\begin{array}{l}\text { Wafers } \\
\text { Lost } \\
\text { at } \\
\text { Dismount }\end{array}$ & $\begin{array}{l}\text { \& Yield } \\
\text { Whole } \\
\text { Wafer }\end{array}$ & Remarks \\
\hline 23 & $\begin{array}{l}226 \mathrm{~B}- \\
102690 \\
\text { CRUSHE }\end{array}$ & 4.842 & 4.846 & $67 \mathrm{~g} / \mathrm{w}$ & $3.672 / 18: 32$ & & & $36 \%$ & \\
\hline 24 & $\begin{array}{l}248 \mathrm{~B}- \\
\text { O22691 } \\
\text { CRUSHE }\end{array}$ & $\begin{array}{r}4.878 \\
D-1\end{array}$ & 4.057 & $67 \mathrm{~g} / \mathrm{w}$ & $3.682 / 18: 03$ & 13 & 12 & $78.6 \%$ & $\begin{array}{l}\text { Mating } \\
\text { Bladepack } \\
\text { to Expt. } 20\end{array}$ \\
\hline 25 & 240 & -0 & & $67 \mathrm{~g} / \mathrm{w}$ & 0 & & Run Aborted & & $\begin{array}{l}\text { cubic boron } \\
\text { nitride would } \\
\text { not cut }\end{array}$ \\
\hline
\end{tabular}


Table III. Details of Bladepacks Used in Slicing Experiments.

\begin{tabular}{|c|c|c|c|c|c|c|c|c|c|}
\hline \multirow{2}{*}{ Expt. } & \multirow{2}{*}{$\begin{array}{l}\text { Blade- } \\
\text { pack } \\
\text { ID \# }\end{array}$} & Dian & & Amount & $\begin{array}{l}\text { Plating } \\
\text { Time }\end{array}$ & \multirow{2}{*}{\multicolumn{2}{|c|}{$\begin{array}{l}\text { Core Dia. } \\
\text { Material } \\
\text { (ins) }\end{array}$}} & \multirow{2}{*}{$\begin{array}{l}\text { Elongation } \\
\text { at Plating } \\
\text { (ins) }\end{array}$} & \multirow[b]{2}{*}{ Remarks } \\
\hline & & Type & $\begin{array}{l}\text { Size } \\
(\mu \mathrm{m})\end{array}$ & $\begin{array}{l}\text { Diamond } \\
\text { (g) }\end{array}$ & $\begin{array}{l}(\operatorname{amp} / \\
\text { min) }\end{array}$ & & & & \\
\hline 1 & $\begin{array}{l}214 A- \\
071090\end{array}$ & $\begin{array}{l}\text { GE } \\
300 \mathrm{~S} \\
\text { Strong }\end{array}$ & 45 & 30 & 1126 & .008 & ss & $.152^{\prime \prime}$ & standard bladepack \\
\hline 2 & $\begin{array}{l}214 \mathrm{~B}- \\
071090\end{array}$ & $\begin{array}{l}\text { GE } \\
300 S \\
\text { Strong }\end{array}$ & 45 & 30 & 1126 & .008 & ss & $.152 "$ & Matching bladepack \\
\hline 3 & $\begin{array}{l}215 A- \\
072590\end{array}$ & $\begin{array}{l}\text { GE } \\
300 \text { S } \\
\text { Strong }\end{array}$ & 45 & 30 & 1066 & $\begin{array}{l}.008 \\
\text { Tung. }\end{array}$ & & -- & Tungsten wire \\
\hline 4 & $\begin{array}{l}215 A- \\
072590-2\end{array}$ & $\begin{array}{l}\text { GE } \\
300 \text { S } \\
\text { Strong }\end{array}$ & 45 & 30 & 1066 & $\begin{array}{l}.008 \\
\text { Tung. }\end{array}$ & & -- & \\
\hline 5 & $\begin{array}{l}215 \mathrm{~B}- \\
072590\end{array}$ & $\begin{array}{l}\text { GE } \\
300 \text { S } \\
\text { Strong }\end{array}$ & 45 & 30 & 1066 & $\begin{array}{l}.008 \\
\text { Tung. }\end{array}$ & & -- & Matching bladepack \\
\hline 6 & $\begin{array}{l}221 B- \\
091390\end{array}$ & $\begin{array}{l}\text { GE } \\
300 S \\
\text { strong }\end{array}$ & 45 & 6 & 1096 & .008 & SS & $.152 "$ & \\
\hline 7 & $\begin{array}{l}228 A- \\
103190\end{array}$ & $\begin{array}{l}\text { GE } \\
300 \mathrm{~S} \\
\text { Strong }\end{array}$ & 60 & 14 & 1575 & .008 & ss & $.152^{\prime \prime}$ & $\begin{array}{l}60 \mu \mathrm{m} \text { diamond; } \\
\text { longer plating time. }\end{array}$ \\
\hline 8 & $\begin{array}{l}228 \mathrm{~A}- \\
103190-2\end{array}$ & $\begin{array}{l}\text { GE } \\
300 \text { S } \\
\text { Strong }\end{array}$ & 60 & 14 & 1575 & .008 & sS & $.152 "$ & \\
\hline 9 & $\begin{array}{l}227 A- \\
103090\end{array}$ & $\begin{array}{l}\text { Natural } \\
\text { Moderat } \\
\text { Friabil }\end{array}$ & & 9 & 1232 & $\begin{array}{l}.008 \\
19\end{array}$ & ss & $.152^{\prime \prime}$ & Natural diamond \\
\hline
\end{tabular}


Table III Continued.

\begin{tabular}{|c|c|c|c|c|c|c|c|c|c|}
\hline \multirow{3}{*}{$\begin{array}{c}\text { Expt. } \\
10\end{array}$} & \multirow{3}{*}{$\begin{array}{l}\text { Blade- } \\
\text { pack } \\
\text { ID \# } \\
227 A- \\
103090-2\end{array}$} & \multirow{2}{*}{\multicolumn{2}{|c|}{\begin{tabular}{lr}
\multicolumn{2}{c}{ Diamond } \\
Type & Size \\
& $(\mu \mathrm{m})$
\end{tabular}}} & \multirow{3}{*}{$\begin{array}{l}\text { Amount } \\
\text { Diamond } \\
\text { (g) }\end{array}$} & \multirow{3}{*}{$\begin{array}{l}\begin{array}{l}\text { Plating } \\
\text { Time } \\
\text { (amp/ } \\
\text { min) }\end{array} \\
\begin{array}{l}1232\end{array}\end{array}$} & \multirow{2}{*}{\multicolumn{2}{|c|}{$\begin{array}{l}\text { Core Dia. } \\
\text { Material } \\
\text { (ins) }\end{array}$}} & \multirow{2}{*}{$\begin{array}{l}\text { Elongation } \\
\text { at Plating } \\
\text { (ins) }\end{array}$} & \multirow[b]{2}{*}{ Remarks } \\
\hline & & & & & & & & & \\
\hline & & $\begin{array}{l}\text { Natural } \\
\text { Moderate } \\
\text { Friabilit }\end{array}$ & $\begin{array}{l}45 \\
\text { ty }\end{array}$ & & & .008 & sS & $.152 "$ & \\
\hline 11 & $\begin{array}{l}227 A- \\
103090-3\end{array}$ & $\begin{array}{l}\text { Natural } \\
\text { Moderate } \\
\text { Friabilit }\end{array}$ & $\begin{array}{l}45 \\
t y\end{array}$ & 9 & 1232 & .008 & SS & $.152^{\prime \prime}$ & \\
\hline 12 & $\begin{array}{l}226 \mathrm{~B}- \\
102690\end{array}$ & $\begin{array}{l}\text { GESTD } \\
\text { Friable }\end{array}$ & 45 & 9 & 1057 & .008 & sS & $.152^{\prime \prime}$ & Friable diamond \\
\hline 13 & $\begin{array}{l}226 \mathrm{~B}- \\
102690-2\end{array}$ & $\begin{array}{l}\text { GESTD } \\
\text { Friable }\end{array}$ & 45 & 9 & 1057 & .008 & ss & $.152^{\prime \prime}$ & \\
\hline 14 & $\begin{array}{l}241 A- \\
11291\end{array}$ & $\begin{array}{l}\text { CDA } \\
\text { Very } \\
\text { Friable }\end{array}$ & $325 / 400$ & 010 & 1232 & .008 & ss & $.152 "$ & $\begin{array}{l}\text { Friable diamond } \\
\text { broader diamond } \\
\text { size distribution }\end{array}$ \\
\hline 15 & $\begin{array}{l}241 A- \\
11291-2\end{array}$ & $\begin{array}{l}\text { CDA } \\
\text { Very } \\
\text { Friable }\end{array}$ & $325 / 400$ & 010 & 1232 & .008 & ss & $.152^{\prime \prime}$ & \\
\hline 16 & $\begin{array}{l}241 B- \\
11291\end{array}$ & $\begin{array}{l}\text { CDA } \\
\text { Very } \\
\text { Friable }\end{array}$ & $325 / 400$ & 010 & 1232 & .008 & ss & $.152^{\prime \prime}$ & \\
\hline 17 & $\begin{array}{l}241 B- \\
11291-2\end{array}$ & $\begin{array}{l}\text { CDA } \\
\text { Very } \\
\text { Friable }\end{array}$ & $325 / 400$ & 010 & 1232 & .008 & sS & $.152^{\prime \prime}$ & \\
\hline 18 & $\begin{array}{l}242 \mathrm{~B}- \\
112291\end{array}$ & $\begin{array}{l}\text { Natural } \\
\text { Moderate } \\
\text { Friabilit }\end{array}$ & $\begin{array}{l}30 \\
t y\end{array}$ & 10 & 924 & .008 & SS & $.152 "$ & $\begin{array}{l}\text { Shorter plating time } \\
30 \mu \mathrm{m} \text { diamond }\end{array}$ \\
\hline 19 & $\begin{array}{l}247 A- \\
022591\end{array}$ & $\begin{array}{l}\text { DuPont } \\
\text { FG }\end{array}$ & 45 & 10 & 1232 & .008 & ss & $.152^{\prime 1}$ & Most friable \\
\hline
\end{tabular}


Table III Continued.

\begin{tabular}{|c|c|c|c|c|c|c|c|c|c|}
\hline$\underset{\#}{\operatorname{Expt}}$ & $\begin{array}{l}\text { Blade- } \\
\text { pack } \\
\text { ID \# }\end{array}$ & $\begin{array}{l}\text { Diamo } \\
\text { Type }\end{array}$ & $\begin{array}{l}\text { nd } \\
\text { size } \\
(\mu \mathrm{m})\end{array}$ & $\begin{array}{l}\text { Amount } \\
\text { Diamond } \\
\text { (g) }\end{array}$ & $\begin{array}{l}\text { Plating } \\
\text { Time } \\
\text { (amp/ } \\
\text { min) }\end{array}$ & $\begin{array}{l}\text { Core } \\
\text { Mater } \\
\text { (ins) }\end{array}$ & $\begin{array}{l}\text { Dia. } \\
\text { rial }\end{array}$ & $\begin{array}{l}\text { Elongation } \\
\text { at Plating } \\
\text { (ins) }\end{array}$ & Remarks \\
\hline 20 & $\begin{array}{l}248 A- \\
022691\end{array}$ & $\begin{array}{l}\text { RVG } \\
\text { Friable }\end{array}$ & 60 & 10 & 1232 & .008 & ss & . $152 "$ & \\
\hline 21 & $\begin{array}{l}248 A- \\
022691-2\end{array}$ & $\begin{array}{l}\text { RVG } \\
\text { Friable }\end{array}$ & 60 & 10 & 1232 & .008 & SS & $.152^{\prime \prime}$ & \\
\hline 22 & $\begin{array}{l}249 A- \\
030791\end{array}$ & $\begin{array}{l}\text { GESTD } \\
\text { Friable }\end{array}$ & 45 & 10 & 1478 & .008 & ss & $.152 "$ & $\begin{array}{l}48 \text { pitch; } 10 \text { degree } \\
\text { included angle. }\end{array}$ \\
\hline 23 & $\begin{array}{l}226 \mathrm{~B}- \\
102690 \\
\text { Crushed }\end{array}$ & $\begin{array}{l}\text { GESTD } \\
\text { Friable }\end{array}$ & 45 & 9 & 1057 & .008 & ss & $.152^{\prime \prime}$ & \\
\hline 24 & $\begin{array}{l}248 B- \\
022691 \\
\text { Crushed-1 }\end{array}$ & $\begin{array}{l}\text { RVG } \\
\text { Friable }\end{array}$ & 60 & 10 & 1232 & .008 & SS & $.152 "$ & \\
\hline 25 & $\begin{array}{l}240- \\
11791\end{array}$ & $\begin{array}{l}\text { Cubic } \\
\text { Boron } \\
\text { Nitride }\end{array}$ & $325 / 400$ & 10 & 1232 & .008 & ss & $.152^{\prime \prime}$ & Cubic Boron Nitride \\
\hline
\end{tabular}


procedure higher cutting rates are obtained at the end when the chord of the cut profile decreases. The average cutting rate for the entire test is lower because of longer time at low initial cutting before the desired cut profile is achieved.

For Experiments 1 and 2 standard plating parameters, diamond size and type were used: Plating thickness $30 \mu \mathrm{m}$ resulting from 1126 Amp minute plating time on $8 \mathrm{mil}$ stainless steel wire, $45 \mu \mathrm{m}$ GE $300 \mathrm{~s}$ strong diamonds with high concentration resulting from charging $30 \mathrm{gms}$ into the plating bath.

Matching bladepacks plated at the same time were used in Experiments 1 and 2. The cutting rate as a function of slicing time is shown in Figures 4 and 5 . Low initial and high final cutting rates are apparent for both tests. It can be observed that the average cutting rate for Experiment 1 was higher than that for Experiment 2 for all the same parameters. The slicing rate for Experiment 2, while not as high as for Experiment 1, was essentially constant for most of the test. This data shows the typical performance of a bladepack in FAST slicing for wafering the first $10 \mathrm{~cm}$ square silicon block. In Experiment 3 tungsten instead of stainless steel wire was used. In this experiment effective slicing was achieved and a high yield of wafers was obtained. The average slicing rate was also high (approximately $125 \mu \mathrm{m}$ per minute), and the same bladepack was utilized for slicing a second silicon block (Experiment 4). It was observed that a high yield of wafers was obtained; however, the average slicing rate for the second block dropped to approximately $82 \mu \mathrm{m}$ per minute. The cutting rate as a function of the slicing time is shown (Figure 6) for Experiments 3 and 4. This shows the characteristic degradation that occurs in slicing multiple blocks with one bladepack. The mating bladepack was used in Experiment 5 and performance in slicing was similar to Experiment 3. The bladepack with tungsten wire performed similar to bladepack 214A\&B made with stainless steel wire. The bladepack used in Experiment 6 was fabricated using the standard stainless steel 


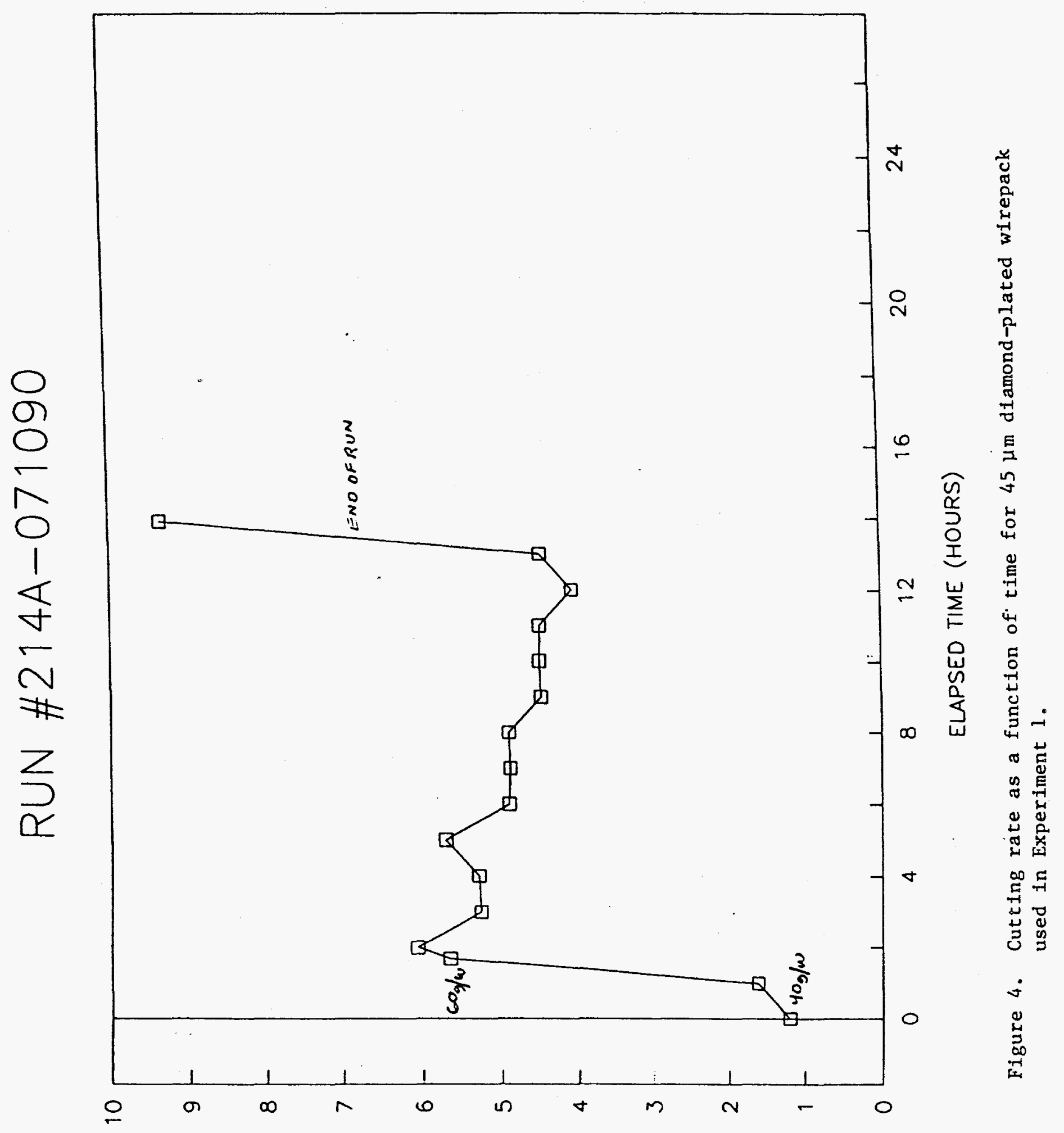

NIW/STIW $\exists \perp \forall Y$ SNILINO 


\section{RUN \#214B-071090}

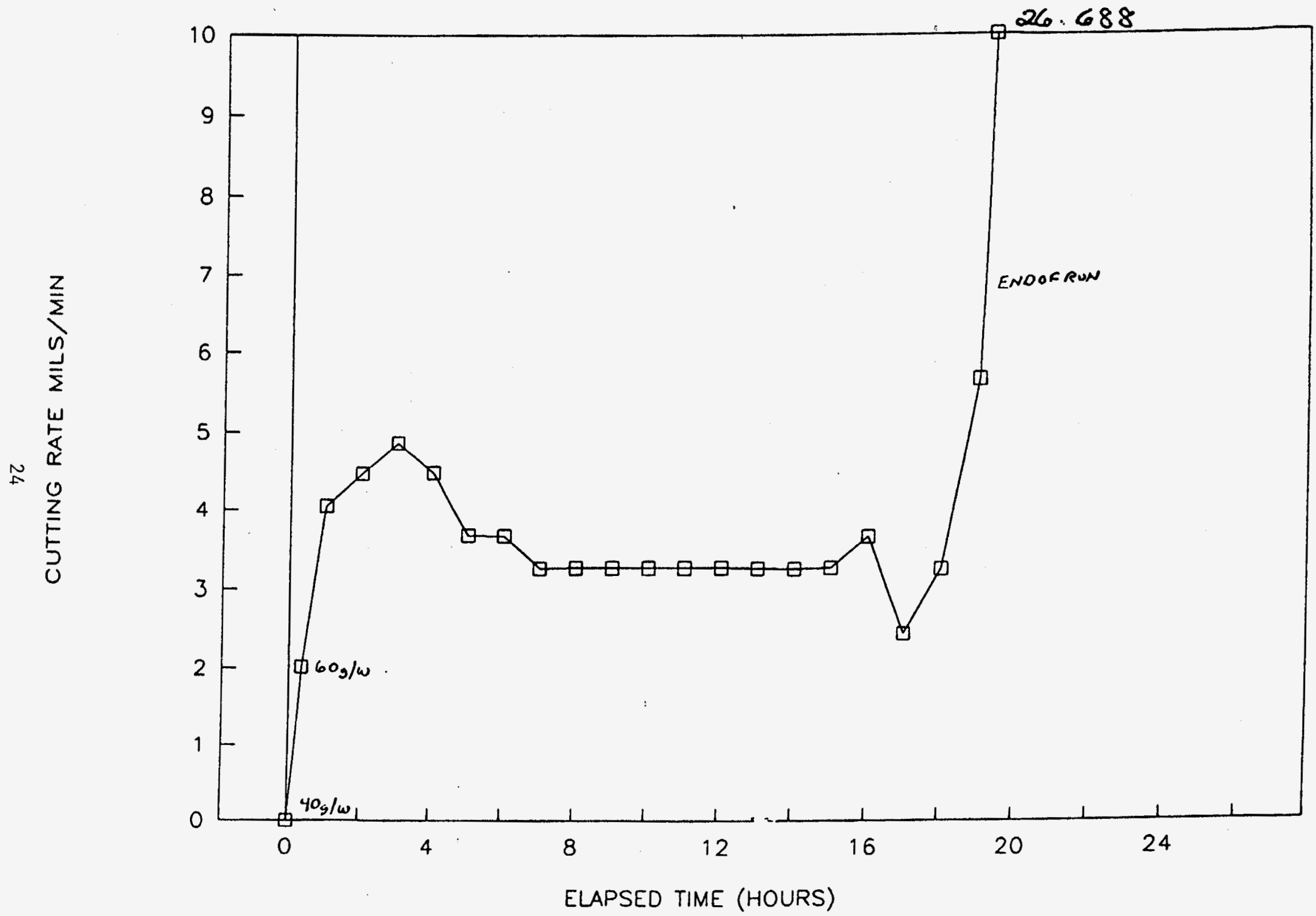

Figure 5. Cutting effectiveness of mating bladepack used in Experiment 1 showing cutting rate with elapsed time during Experiment 2. 


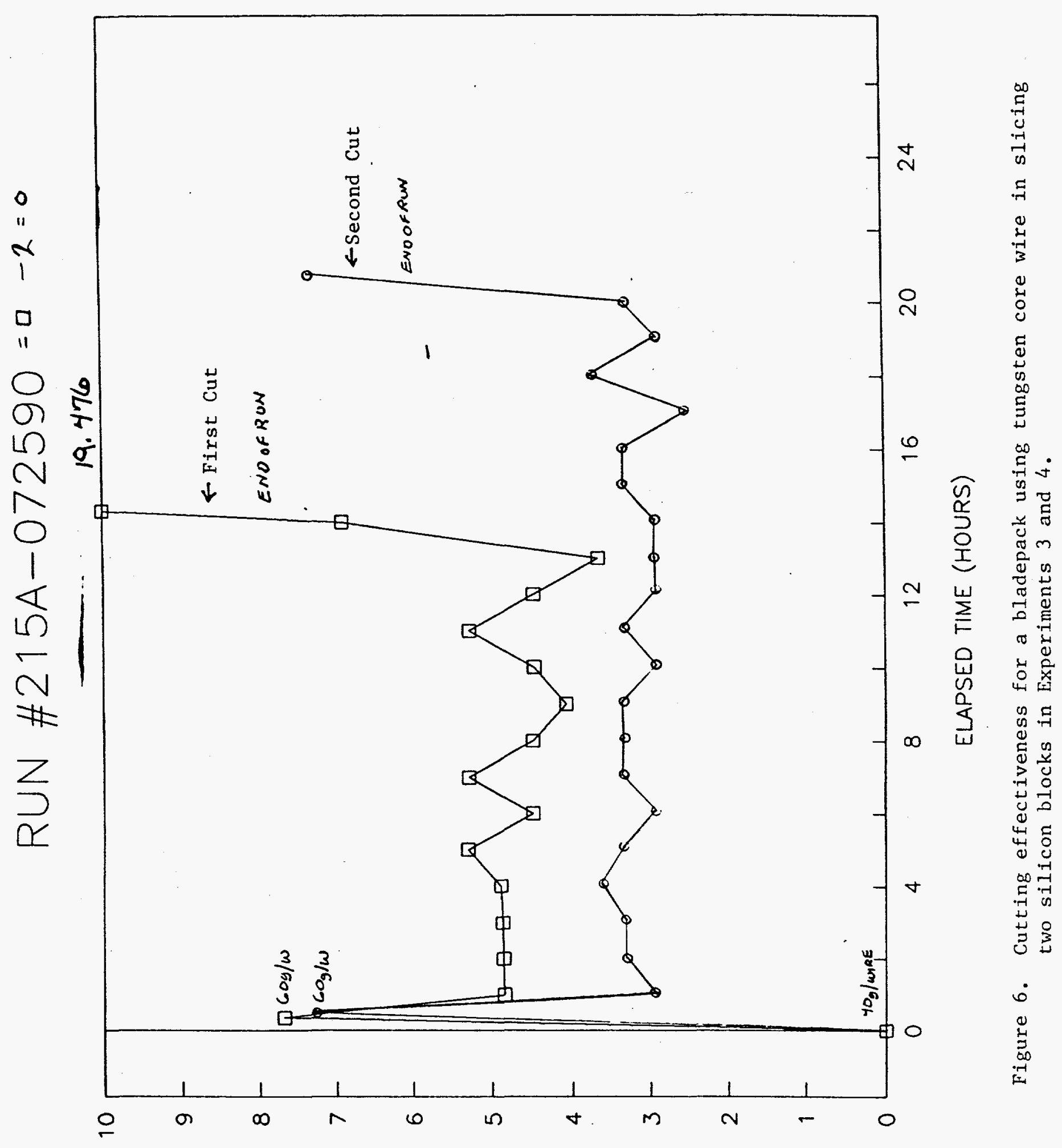

NIW/STIW $3 \cdot \cdot 4$ ONILINO 
core wire, and the diamonds charged to the plating bath was less compared to earlier bladepacks fabricated. The slicing test showed a similar result for slicing the first silicon block showing diamond concentration is not an important factor.

In Experiment 760 micron diamond was used and the feed force was increased quickly to 67 grams per wire. It was observed that some chipping of the silicon occurred, and these chips were caught between the wires causing major destruction of the wafers. This run was therefore aborted. The same wirepack was used in Experiment 8 and the usual procedure of increasing feed force in small increments was followed. Effective slicing was achieved; however, the average cutting rate was less than $75 \mu \mathrm{m}$ per minute.

For Experiment 9 the diamond type was changed from man-made to natural $45 \mu \mathrm{m}$. High initial cutting rates (greater than 125 micron per minute) were obtained, and the block was sliced with overall cutting rates greater than 100 $\mu \mathrm{m}$ per minute with a wafer yield of 92.5 percent. The same bladepack was used in Experiment 10; however, after slicing for approximately 13 hours, the test was aborted as wire wander was observed. Wander usually is associated with poor cutting. The same bladepack was dressed after this experiment and utilized for slicing in Experiment 11. The initial cutting rates in Experiment 11 were significantly higher than the cutting rates towards the end of Experiment 10. This shows that the bladepack can be revived for effective slicing by dressing.

The bladepack used for Experiments 12 and 13 was electroplated with friable diamonds. Effective slicing was achieved for slicing two blocks of silicon with high yields (96 and 87 percent). The bladepacks used for Experiments 14 through 17 were electroplated using a more friable type of diamond (CDA) which has a 325 to 400 grit size distribution due to a separation process instead of a micronization process that results in a tighter size distribution. Each 
bladepack was used to slice two silicon blocks; however, the cutting rate for slicing the second block and the yield of wafers was low. This data showed that GESTD-type friable diamond performs better than the CDA-type diamonds for FAST slicing. In Experiments 19 through 20 different kinds of diamonds (DuPont FG, RVG friable and GESTD friable) were evaluated for FAST slicing. In run 18 natural $30 \mu \mathrm{m}$ diamond was used and plating time was shortened from 1232 to 924 Amp minutes. The cutting rate of this bladepack degraded more rapidly than for $45 \mu \mathrm{m}$ natural diamond with thicker plating. This is a very interesting effect which may be due to the smaller diamonds or shorter plating time which would result in thinner nickel plating that would not hold the diamond as tenaciously.

Experiment 19 was conducted with $45 \mu \mathrm{m}$ explosively formed DuPont diamond. It. is a polycrystalline diamond that is very weak and is often used for polishing because it breaks down so easily. The diamond has many impurities that caused nickel nodules to form on the plating and required dressing to expose the diamond tips. The cutting rate was low, $1.59 \mathrm{mils} / \mathrm{minute}$, but it did not degrade during the run. The nickel plating may have provided the diamond from cutting effectively. Experiment 20 was conducted with a bladepack plated with $60 \mu \mathrm{m}$ friable diamond. It performed better than the bladepack used in Experiments 7 and 8 that used a stronger $60 \mu \mathrm{m}$ diamond. Experiment 21 was performed with the same bladepack and still had good cutting rate at the end of cutting the second bar. Experiments 23 and 24 were performed with bladepacks that were crushrolled to position many diamonds at the same height. The bladepack used in Experiment 12 ( $45 \mu \mathrm{m}$ friable diamond) was crush-rolled and reused in Experiment 23 to achieve nearly the same cutting rated achieved during the first cut (Experiment 7). This is an extremely interesting result since a high constant cutting rate was maintained throughout the run. The cutting rate of Experiment 
24 was similar to Experiment 20 where the matching bladepacks $(60 \mu \mathrm{m}$ friable diamond) was used after crush rolling.

The data showed the following:

(1) Friable diamond performed better than natural type, and among the friable diamonds the RVD-type diamonds showed the best performance.

(2) New and used bladepacks that were crush-rolled exhibited almost no degradation from the beginning to the end of the cut.

(3) Cutting rates can be increased by dressing.

(4) Diamond concentration and type of core wire used had no noticeable effect on cutting performance.

\subsection{Analysis of Data}

From the above slicing tests it can be summarized that the bladepacks lose cutting effectiveness with time. The cutting effectiveness can be revived by dressing; however, the dressing operation can only be carried out after slicing a block. Examination of the wires after the wirepack had lost cutting effectiveness showed that the diamond concentration on the wires was still high, and there was no observable diamond pullout. It can be concluded that diamond pullout is not the failure mechanism for the wirepacks. It was also observed that bladepacks fabricated with 30 grams and 6 grams of diamond charged to the plating bath showed similar performance. Therefore, it can be concluded that the diamond concentration being low is not responsible for loss in cutting effectiveness.

Among the various diamond types evaluated, it was observed that the friabletype diamonds performed better than the stronger synthetic, blocky or natural type. SEM examination of the diamonds is shown in Figure 7. This is contrary to expectation because the friable-type diamonds are not as hard and can fragment under high loads. From this data it can be concluded that a very small number 

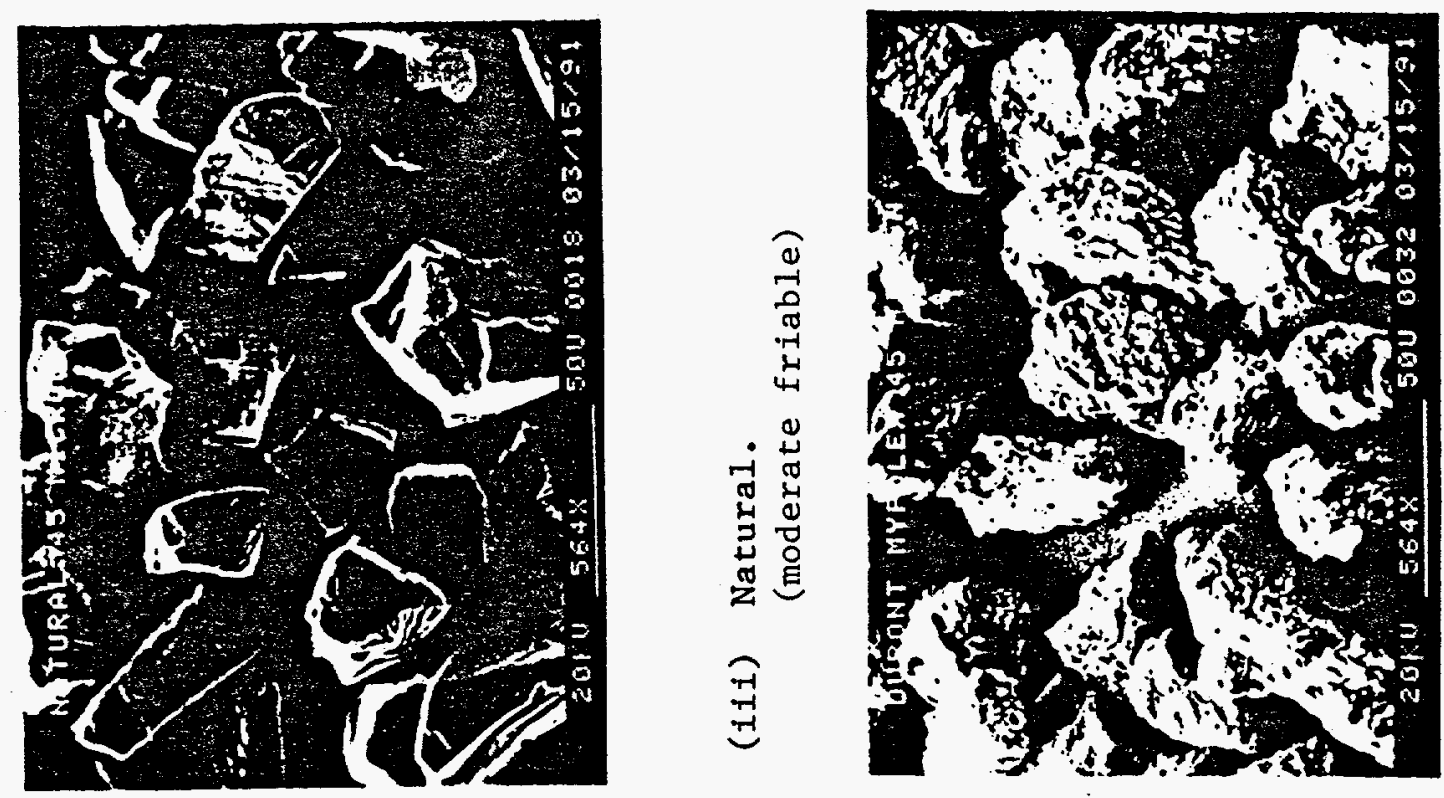

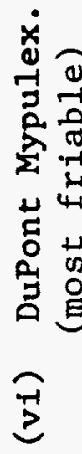
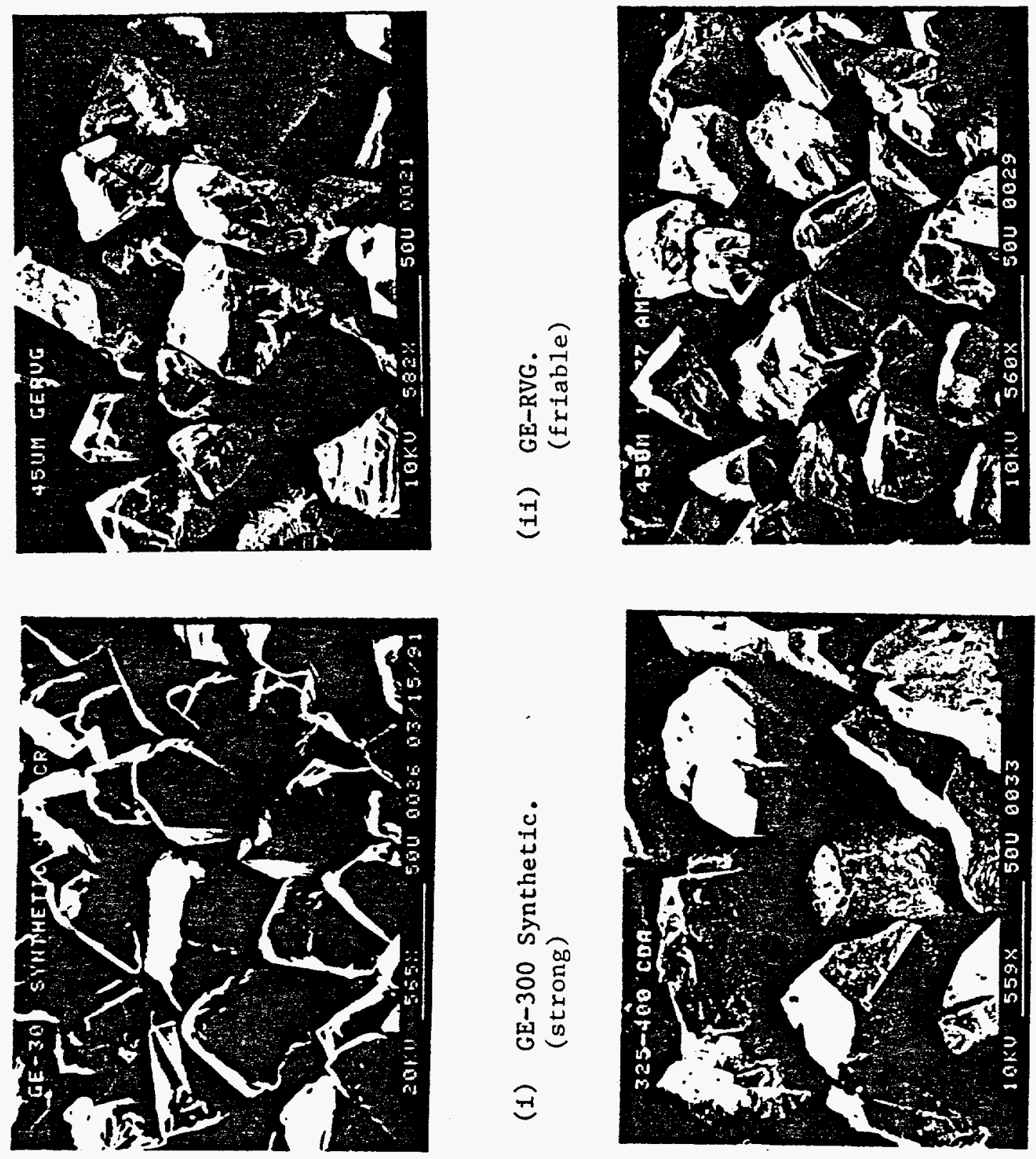

是

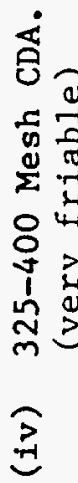


of diamonds on the wire contact the silicon workpiece and are used for slicing. This can happen only if the tips of the diamonds are at different elevations on the wire and the tips at highest elevations prevent other diamonds at lower elevations from contacting the workpiece. If the number of diamonds contacting the workpiece at any time is small, the feed force on each diamond tip is high. Under these conditions the diamond tip may become blunt by forming a "wear flat" or it may be moved in its nickel setting to a less favorable cutting orientation during the reciprocating action. If wear flats are formed on most of the diamonds at highest elevations and no new diamonds contact the workpiece, then the effective pressure on the wear flats will become less as slicing progresses and the wear flat increases in size. Movement of a diamond into a less favorable cutting orientation would involve rotating from a point to a flat surface as a result of the large cutting forces and the push-pull action associated with reciprocating cutting. Wear flats and/or movement of the diamond from a point to a flat explain the observation that the slicing effectiveness of bladepacks decreases as slicing progresses. Since the effective pressure on the diamond tip decreases as the flat increases in size, the diamond cannot be removed from the wire so diamonds at lower elevations contact the workpiece; effective slicing cannot be restored. Since the number of diamonds at the highest elevation is very small compared to the total number of diamonds, correlation was not observed for the amount of diamonds charged to the plating bath and slicing effectiveness. Once a silicon block is sliced, the diamonds at the highest elevation can be removed by dressing so that sharp diamonds at lower elevations contact the workpiece. Previous experiments have shown that slicing effectiveness of the wirepack can be restored by dressing. Once again the dressing operation removed very few diamonds; therefore, cutting effectiveness was always restored after dressing irrespective of the amount of diamonds charged to the plating bath. 
Attempts were made to observe different elevations of diamonds on the wires, but proof could not be obtained with optical microscopy or even with Scanning Electron Microscopy (SEM). To confirm the hypothesis that diamonds are at different elevations and that very few diamonds contact the workpiece, an experiment was carried out using a flat plate. This plate was electroplated with diamonds similar to those utilized for electroforming of wires. Sections of this plate were examined with an SEM, and it was observed that high concentration of diamonds were obtained. A representative sample is shown in Figure 8 . In this figure the marker at the bottom represents $50 \mu \mathrm{m}$. It can be seen that the $45 \mu \mathrm{m}$ diamonds have different length-to-width ratio; therefore, the position of the diamond affects its elevation from the plate. Some of the diamonds appear to be buried under the plating while others have a substantial portion above the plating. A section of the plate was tilted about $20^{\circ}$ to observe the elevation as shown in Figure 9. This figure indicates that the diamonds have different elevations above the plating. A lower magnification picture (Figure 10) shows that the diamond concentration was very high. The diamond plated plate was rubbed across a silicon piece at high feed forces ( -10 times that utilized for FAST slicing). The silicon block was examined with an SEM and five consecutive sections of scratches are shown in Figure 11. It can be observed that the grooves formed on the silicon bar are at different spacing with different depth for each groove. It is important to note that the marker for Figure 11 is $50 \mu \mathrm{m}$, similar to that in Figure 8 in which the diamond concentration was shown. This confirms that even under very high feed forces, the number of diamonds contacting the workpiece is a small fraction of the total number of diamonds. The diamonds contact the workpiece at different elevations therefore the feed force on the diamonds varies and grooves of different depth are formed. A calculation based 


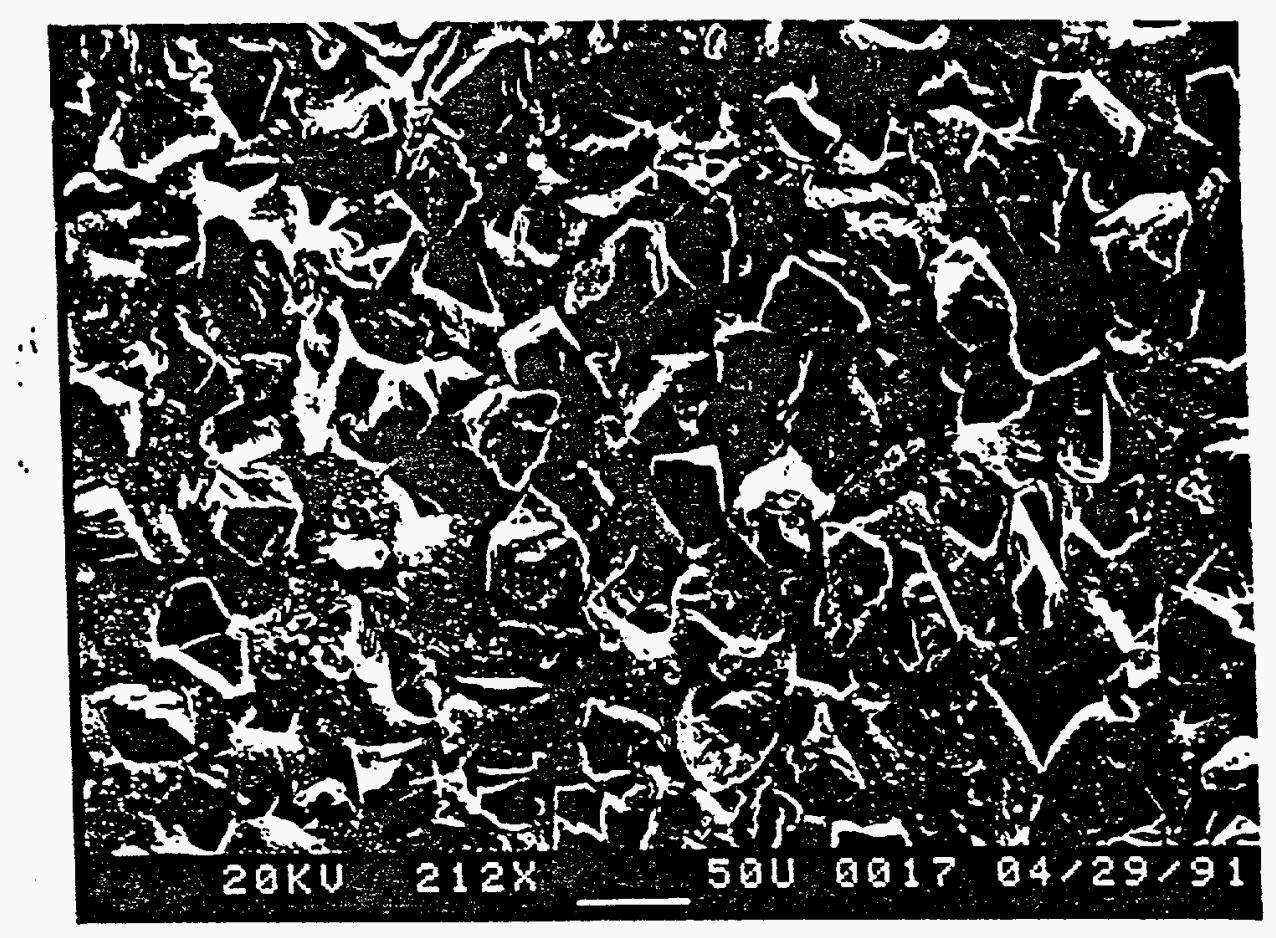

Figure 8. Examination of diamond-plated wire showing high concentration of diamonds.

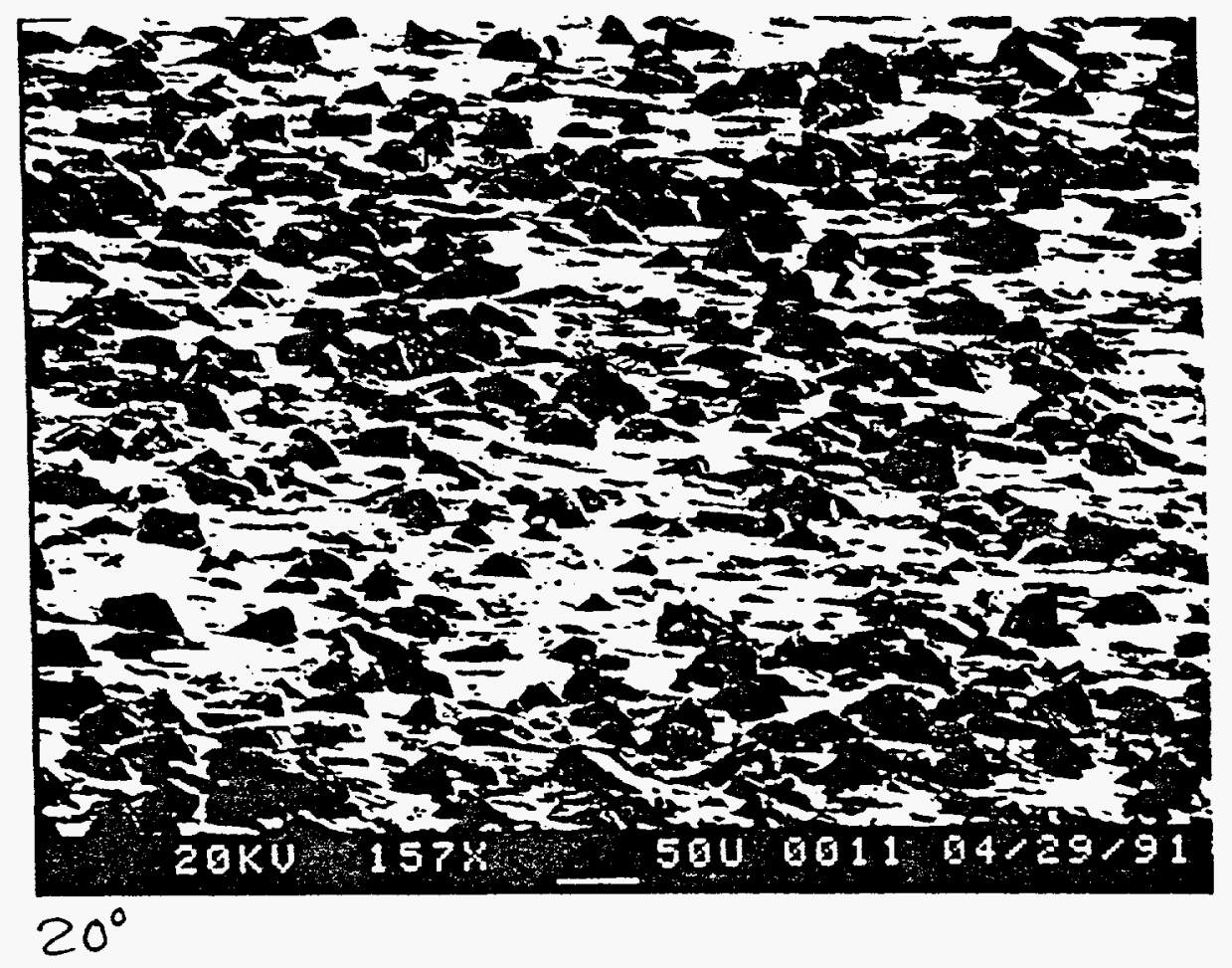

Figure 9. Examination of a diamond-plated wire section tilted about $20^{\circ}$ to observe the elevation of diamonds. 


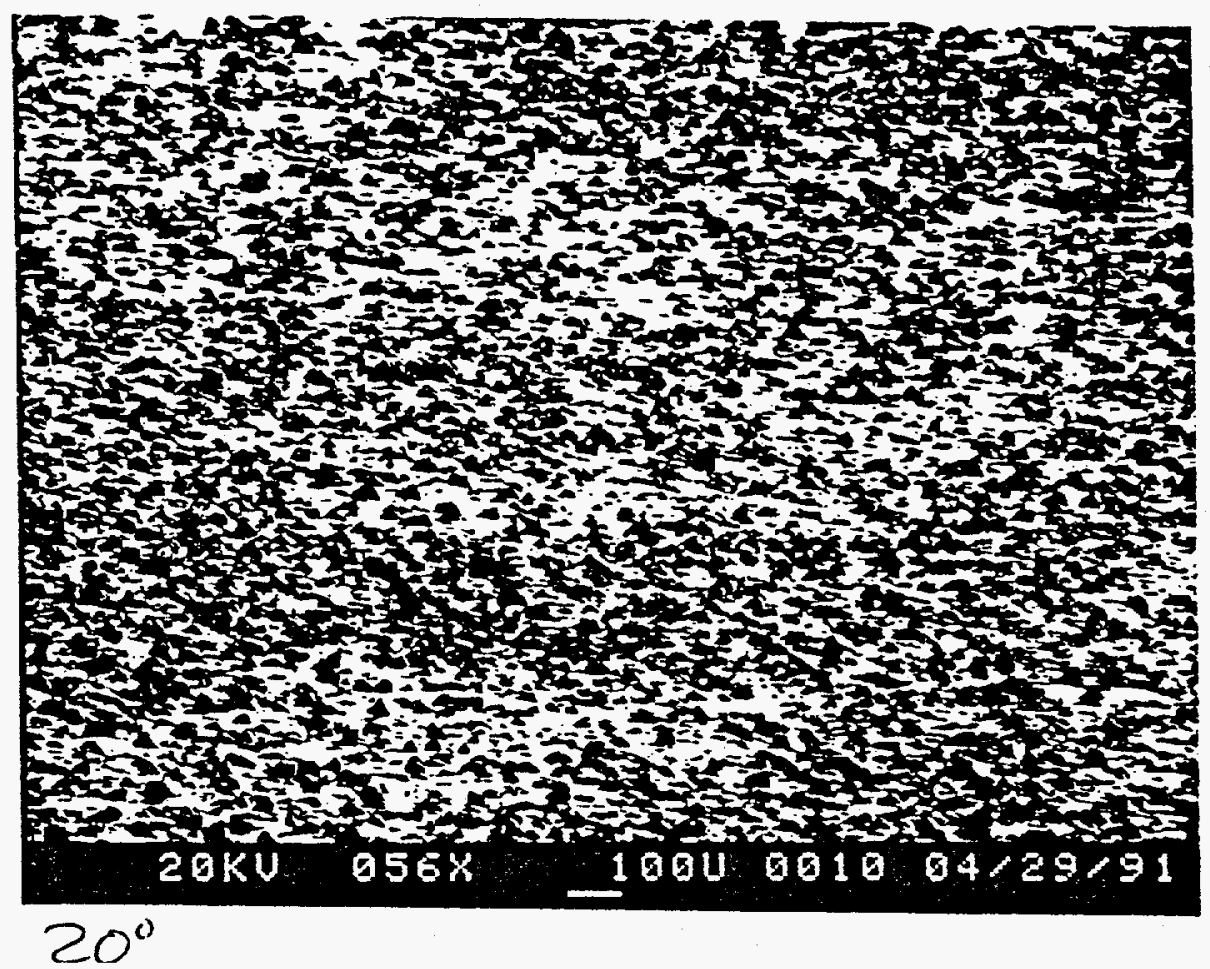

Figure 10. A low magnification examination of a diamond-plated wire showing high diamond concentration. 


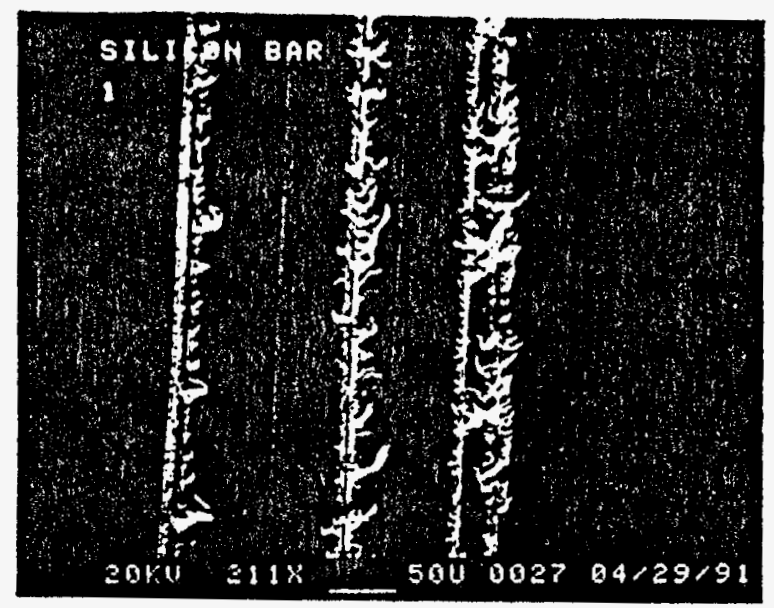

(i)

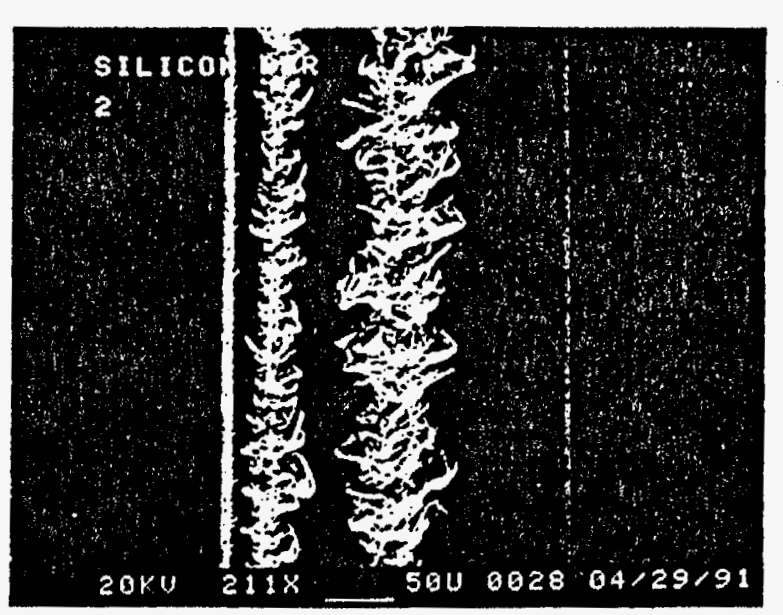

(ii)

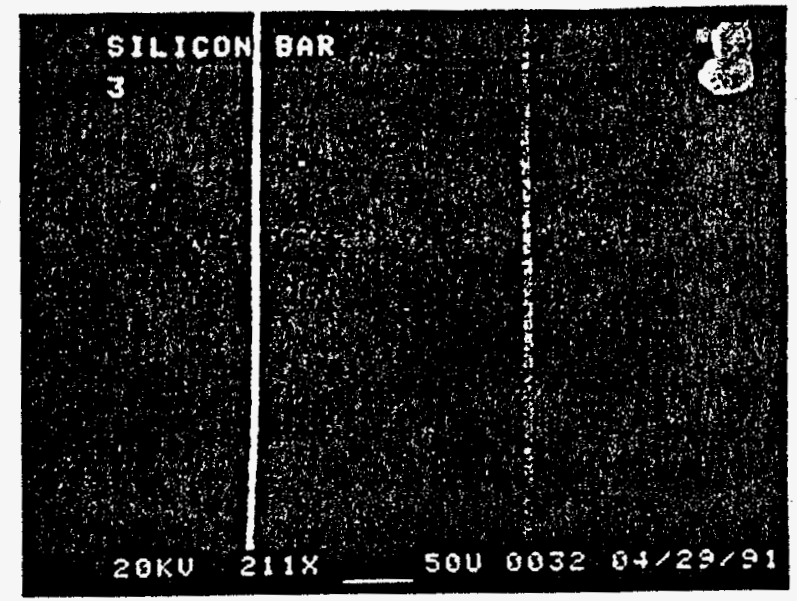

(iii)

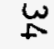

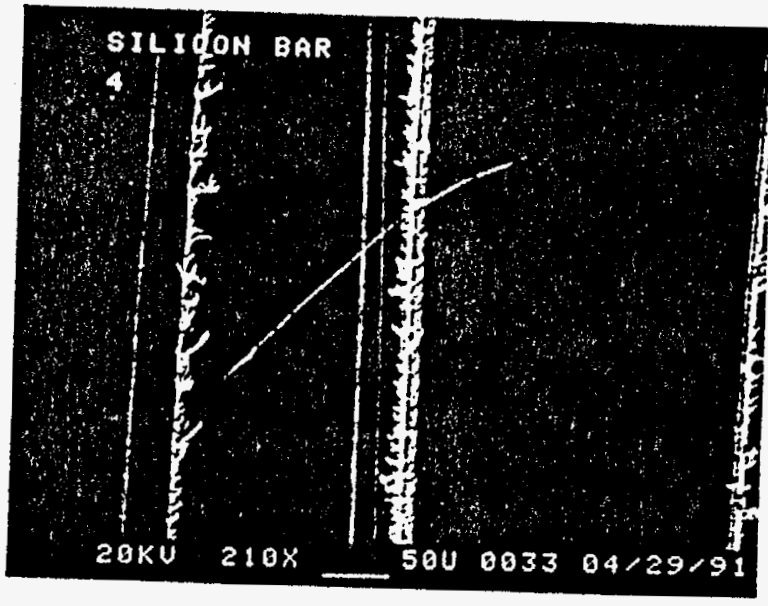

(iv)

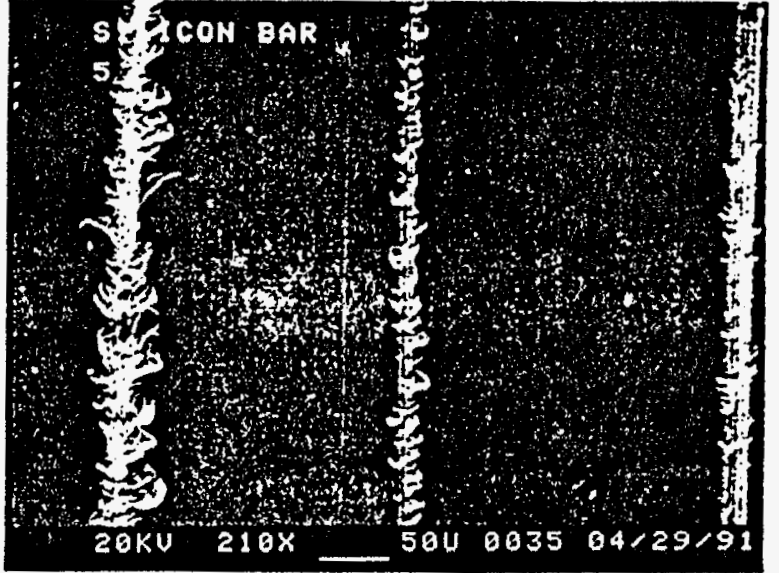

(v)

Figure 1.l. SEM examination of five consecutive sections of silicon block showing scratches after abrasion with diamond-plated plate. 
on SEM examination of the total number of diamonds and scratches formed showed that approximately 0.18 of the available diamonds contact the workpiece.

An attempt was made to observe wear flats on diamonds on wires after slicing of silicon blocks. As seen from Figure 8 and a magnified view of a representative diamond in Figure 12, the diamonds show faceting, and it was difficult to distinguish between the original facets and wear flats formed. Characterization was made even more difficult because after use it was difficult to identify the diamonds which had contacted the workpiece during slicing as only 0.18 of the diamonds were involved in slicing. Another experiment was therefore carried out to distinguish the diamonds involved in slicing from the total number of diamonds. A similar diamond plated plate discussed earlier was coated with a thin film of gold by evaporation. This plate was vigorously rubbed across a silicon block under high feed forces. Examination of this plate using Robinson Back-Scattered Electron Imaging (RBEI) is shown in Figure 13. The areas of diamonds contacting the silicon block had abraded the gold film and can be seen as dark spots due to charging from the electron beam. Again it can be seen that the number of diamonds contacting the silicon block is a small fraction and that among them each of the diamonds has a different area of contact.

These experiments confirm the hypothesis that very few diamonds contact the workpiece during slicing and that the effective feed forces on each diamond varies during FAST slicing so that the cutting rate varies during slicing. When the number of diamonds contacting the workpiece is small, the effective feed forces at the diamond tip is high which may result in formation of wear flats or rotation of a diamond tip to a flat. When the number of diamonds contacting the workpiece is higher, wear flats or diamond rotation may be minimized as observed with bladepacks that were crush-rolled to achieve a more uniform height of diamond tips. The degradation of the wires may be dependent on this 


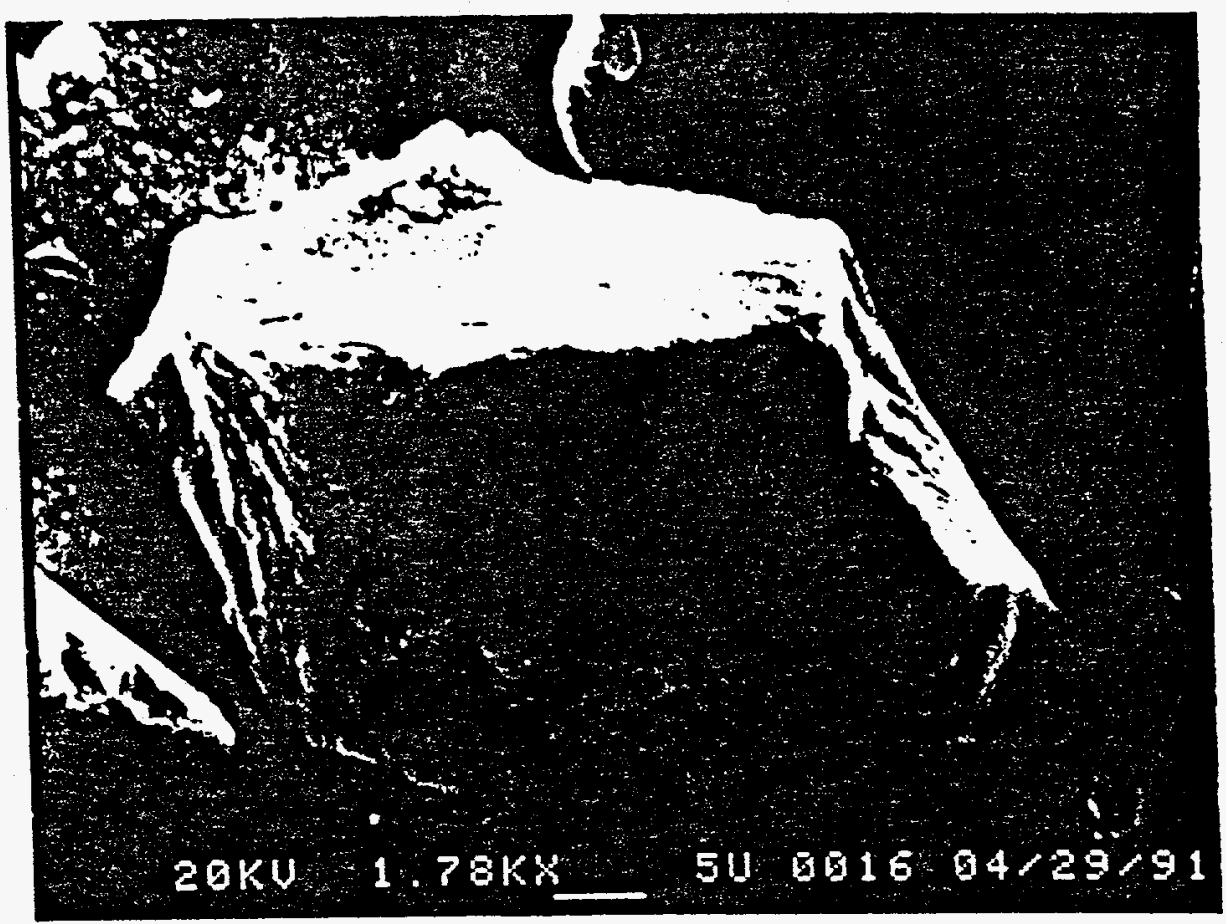

Figure 12. A magnified view of a $45 \mu \mathrm{m}$ diamond showing faceted faces.

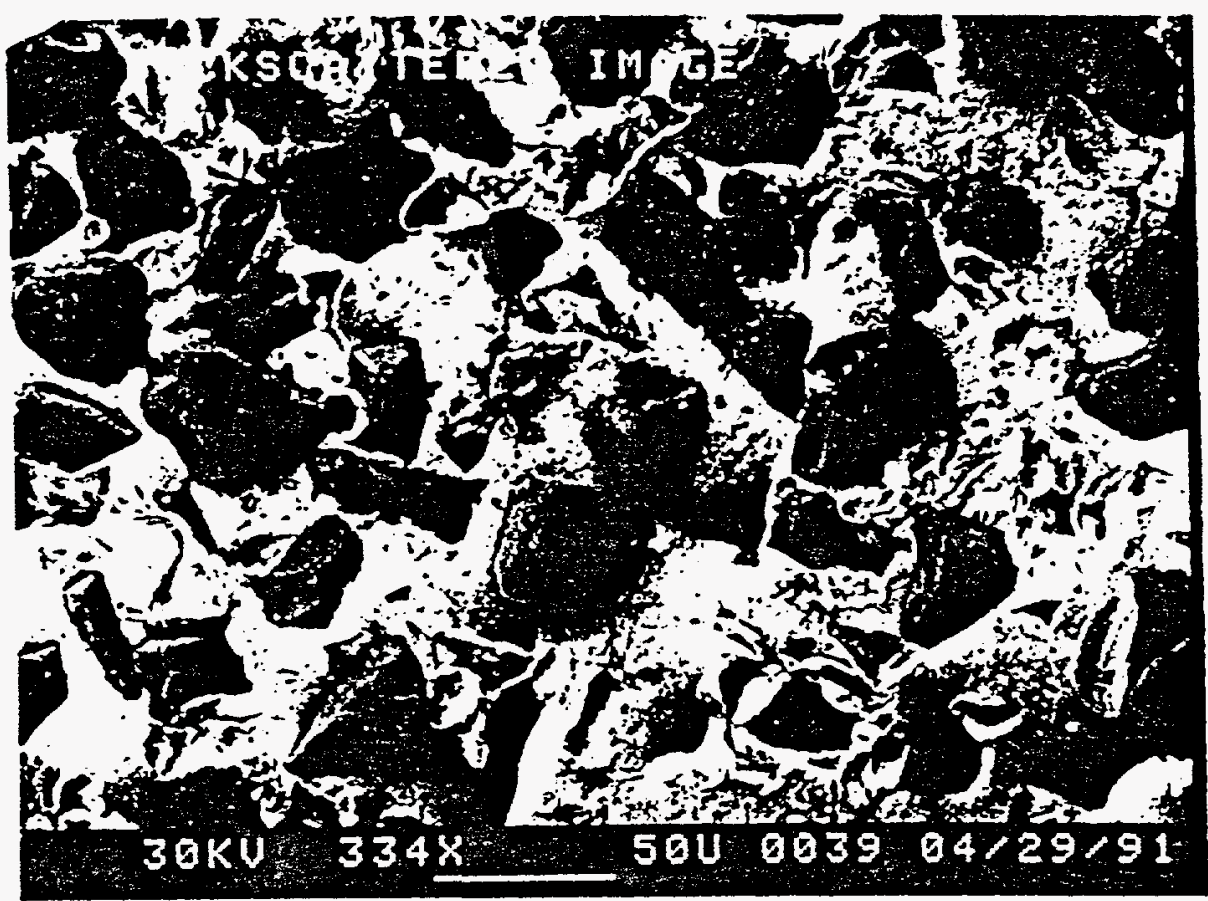

Figure 13. RBEI examination of a diamond-plated plate after vigorous rubbing showing that only a few diamonds were contacting the workpiece. 
parameter; however, slight variation in the length-to-width ratio for each diamond could cause significant variation in the elevation of the diamonds after plating. Crush-rolling may be necessary to equalize the diamond tip height and replating may be needed to prevent diamond movement after crush-rolling.

\subsection{Dry Cutting}

Considerable work has been done in the industry on wear of diamond grinding quills $\mathrm{s}^{12-15}$. The relationship between grinding and abrasive wear has also been correlated. It has been reported ${ }^{16}$ that the ultimate performance of both grinding and abrasive wear depends on the abrasive grits of the tools rather than differences in construction of the tools. Similar to abrasive wear, in abrasive slicing the efficiency of the action of individual abrasive particles is influenced by the hardness of the material being sliced and the rate of ploughing into the material. In the case of FAST slicing of silicon, therefore, the individual shape and morphology of diamond particles and their penetration into the silicon will strongly influence the efficiency of slicing. A theoretical analysis is presented in section 3.4 .

A comparison of wet versus dry grinding ${ }^{17,18}$ and the effect of temperature ${ }^{19}$ on grinding has been reported in literature. It is believed that the performance of grinding wheels is improved by using coolant. Dramatic increase in the wear rate of quills was observed when coolant was applied at low speeds, but only a modest increase in wear rate was observed when it was applied at higher speeds ${ }^{18}$. Applying this data to FAST slicing using coolant, the wear rate of the diamond should be high when slicing is carried out at low tool speeds and wear flats on diamonds minimized without use of coolant.

The problems involved in dry slicing are the removal of silicon swarf during slicing and the graphitization of diamond if the temperature at the 
diamond tip is very high, as observed with ID slicing when there is no adequate coolant.

An experiment was carried out to evaluate dry FAST slicing. An electrostatic field was set up near the silicon block so that the submicron swarf particles were removed from the area of slicing. Initially coolant was used to establish the cutting rate, after which the coolant was stopped and the electrostatic field applied. This field was effective in removing some of the swarf and the rest of the swarf was removed with a vacuum. It was observed that the slicing rate was considerably lower with dry slicing to a point where effective slicing was not achieved. At that point the electrostatic field was switched off and the coolant was applied. The original cutting rate was restored.

This experiment showed that dry slicing is not effective with FAST and graphitization of diamond, if any, is minimal. This may be because the effective tool speed is low and the effective pressure at the diamond tip is also low. In conventional grinding and abrasive cutting, tool speed is low but pressure is high. For abrasive cutting (such as ID slicing) the tool speed and the pressure are both high; therefore, dry cutting cannot be utilized because of graphitization of diamonds.

\subsection{Correlation of Experimental Data with Theoretical Analysis}

\subsubsection{Diamond Size Distribution}

Diamonds obtained from vendors are classified according to size; however, there is a spread of diamond sizes for each size specified. In addition, the length-to-width ratio for diamonds will vary. The diamonds obtained for plating of wires are specified for a tight distribution. A typical distribution of a $45 \mu$ diamond batch is shown in Figure 14. This shows that the geometric mean size is $46.07 \mu$ with a standard deviation of 7.781 and a median size of $46.24 \mu$. 
Date: Thu Feb 191991 11:29:35 a.w. Oper ID: 0 \{auple luuber: 0 Stop type: TIKE Elapsed tiae (sec): 22.6 Blockaqes: 0

geoaetric wean size $=\mathbf{4 6 . 0 7}$

standard deviation $=7.781$

sedian size $\quad=46.24$ arithwetic mean size $=46.72$

coeff.of variation $=36.89 \quad$ skewness $=-6.026 \mathrm{e}-002$

sode size $\quad=46.54$

Tots =

51269

Lon at $28 \quad 25.12$

0 High at 9077.84

1 Top of scale is

2400

Sraph of DIhK Size v8. Differential Counts Fros channel

28 to 88 skip: 2

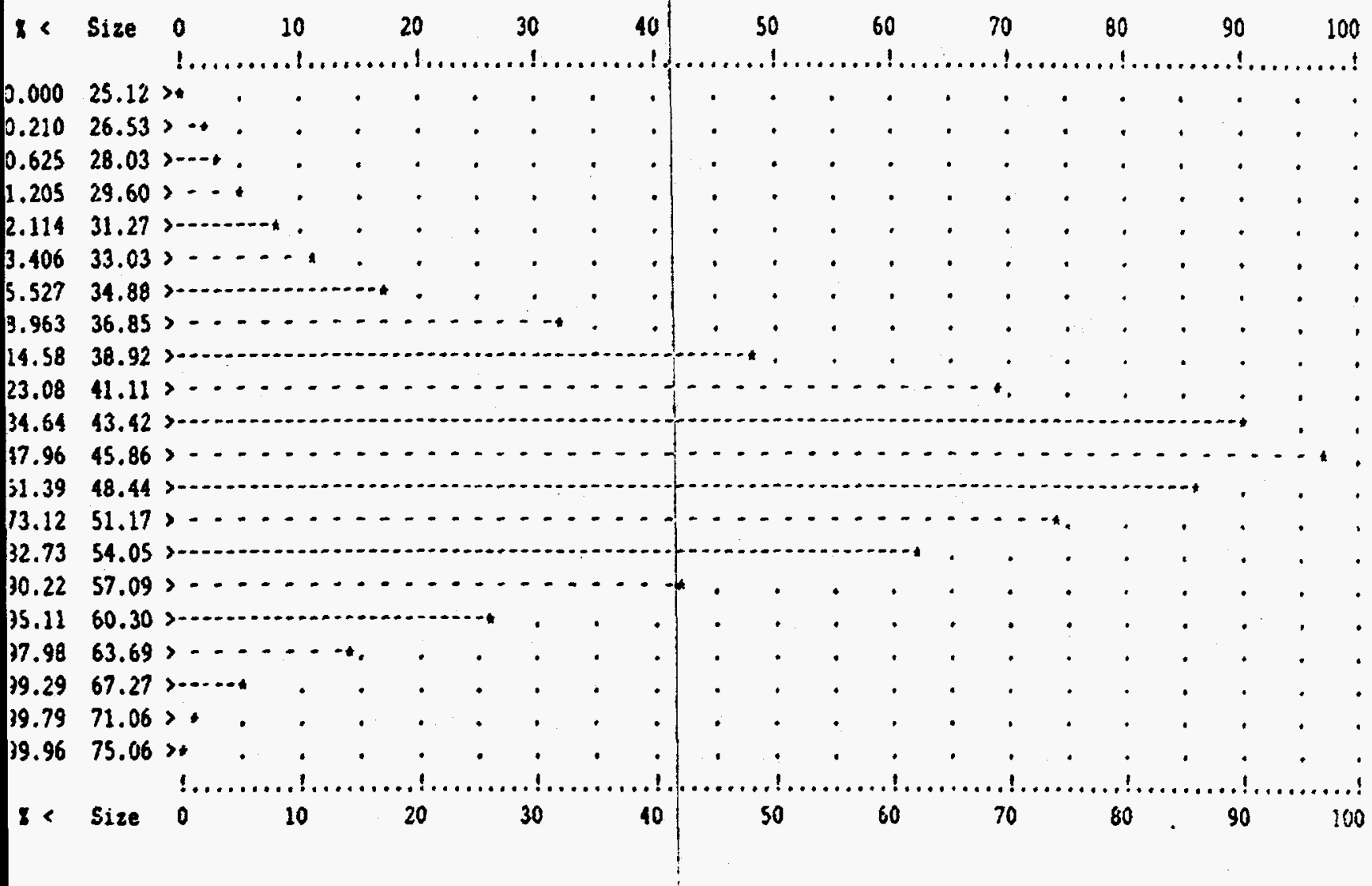

Figure 14. A typical distribution of $45 \mu \mathrm{m}$ diamonds. 
For the diamond size, $a$, if there are a total of $n$ diamonds, then within a small diamond size, da, the corresponding number of diamonds, dn, can be expressed as follows:

$$
\mathrm{dn}=\mathrm{n} \cdot \underline{\mathrm{da}}
$$

or

$$
\frac{\mathrm{dn}}{\mathrm{da}}=\frac{\mathrm{n}}{\mathrm{a}}
$$

From the data in Figure 14, it can be seen that the maximum number of diamonds are $45.86 \mu$ size; however, there are also some diamonds up to $75 \mu$ size. The plating thickness to hold the diamonds is typically $30 \mu$ so that approximately $2 / 3$ of the $45 \mu$ diamond is held in the nickel plating. The largest diamonds will not be held with the plating, and during initial slicing these diamonds will be removed. Diamonds of approximately $60 \mu$ size will be held and about half the diamond held in the plating; therefore, it can be assumed that diamonds larger than $60 \mu$ size will not remain in the slicing operation due to pullout during initial slicing. Of the diamond sizes remaining, the larger diamonds protrude farther than the smaller diamonds and contact the silicon workpiece during slicing.

If it is assumed that the diamond penetrates approximately $3 \mu \mathrm{m}$ into the workpiece at feed forces used in FAST slicing, then only diamonds of 56 to $60 \mu$ size will contact the silicon workpiece. From Figure 14 it can be seen that approximately 5 percent of the total number of diamonds $\left(n_{0}\right)$ are of 57 to $60 \mu$ $(\mathrm{da}=3)$ size. Therefore,

$$
\frac{\mathrm{dn}}{\mathrm{da}}=\frac{0.05 \mathrm{n}_{0}}{3}=0.017 \mathrm{n}_{0}
$$

This means that less than 2 percent of the total diamonds plated on the wire would be affective for FAST slicing, and the remaining diamonds would not contact the workpiece. 
The above analysis utilizes only the distribution of diamond sizes to conclude that less than 2 percent of the available diamonds contact the silicon workpiece during slicing. Other variables such as shape factor (length-towidth ratio) of the diamonds, orientation of the largest dimension of the diamonds with respect to the wire, the curved surface of the wire, etc., will also affect the number of diamonds contacting the workpiece during slicing. As a first approximation it is expected that these factors would reduce the number of diamonds contacting the workpiece as compared to those obtained from equation (3). This is consistent with the experimental observation that less than 1 percent of the available diamonds contact the workpiece during FAST slicing. In view of this, equation (3) can be utilized as a probability factor, since these diamonds are not removed due to pullout so that the next lower size diamonds can be involved in slicing. If these diamonds are removed so that the next layer of diamonds is exposed, then the number of diamonds contacting the workpiece for the same penetration in the workpiece would be considerably higher because for each successive da -3 , the percentage of diamonds increases until the geometric mean size of $46 \mu$ is achieved.

In general for a penetration depth of diamond into the silicon workpiece of $h$, the number of diamonds with penetration between $h$ and $(h+d h)$ can be given by the following equation:

$$
\frac{\mathrm{dN}}{\mathrm{dh}}=\underset{\mathrm{gN}}{\mathrm{g}} \underline{\underline{h}}
$$

Where $g$ is a geometric factor $(-0.02), N_{0}$ is the total number of diamonds on the wire and $a$ is the median size of diamonds. Integration of equation (4) shows that the total number of diamonds, $N$, involved in slicing with a penetration depth of $\mathrm{h}$ can be given by:

$$
N=\frac{g N_{0}}{2 a}
$$


This shows that $\mathrm{N}$ is a quadratic function of $\mathrm{h}$; for small increases in $\mathrm{h}$, large increases in $\mathrm{N}$ will be obtained. This is consistent with data in Figure 14 which

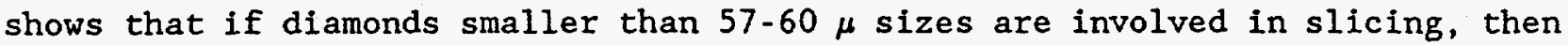
the number of diamonds contacting the workpiece will increase considerably.

The above analysis also shows that as the concentration of diamonds on the wire, $N_{0}$, is increased, it will have a smaller effect on $N$ as compared to increasing the penetration depth, h. Therefore, no difference in slicing effectiveness was observed for bladepacks fabricated using $30 \mathrm{~g}$ or $6 \mathrm{~g}$ of diamonds charged to the plating bath.

\subsubsection{Penetration of Diamonds Into the Workpiece}

From the experimental data it can be concluded that very few diamonds are contacting the silicon workpiece during FAST slicing. This data can be confirmed with theoretical analysis based upon diamond size distribution. A small fraction of the total diamonds protruding farthest above the plating contact the workpiece, and other diamonds which are slightly below cannot contact the sïlicon during slicing. If more diamonds should be involved in the slicing, then it should be possible to accomplish this using two approaches. In one approach conditions should be developed so more diamonds protrude equally above the nickel plating so all diamonds contact the workpiece. In the second approach if the penetration depth of diamonds into the silicon workpiece can be increased, the diamonds which are slightly above the silicon workpiece during FAST slicing will penetrate the silicon and be involved in the slicing. Experiments were performed to confirm these approaches experimentally, and the results are discussed below.

\section{Increasing Diamonds With Same Protrusion}

An experiment was carried out to evaluate if, when an electroplated bladepack is crushed between rollers, a larger number of diamonds can be 
positioned so the average protrusion above the plating is similar. A bladepack which had been used for slicing two silicon blocks (in Experiments 12 and 13) was placed between flat rolls and pressure was applied. One of the wires was examined after crushing using SEM. Two views of this wire are shown in Figure 15. It can be seen that the flat rollers squeezed the nickel diamond matrix flat on the cutting edge of the wire. This wire still has a high concentration of diamonds and after crush-rolling the diamonds on the surface in contact with the rolls, appear to have similar protrusion.

To confirm that this approach would revive the cutting effectiveness, this bladepack was used to slice a $10 \mathrm{~cm}$ silicon block in Experiment 23 (Table II). It was observed that the initial cutting rate for this experiment was 4.842 mils per minute, compared to $3.649 \mathrm{mils}$ per minute at the end of slicing prior to the crushing of the bladepack. The cutting rate was maintained throughout the slicing experiment and the average cutting rate for this block was $3.672 \mathrm{mils}$ per minute, considerably higher than the 2.854 mils per minute achieved for the block prior to crushing. In Experiment 23 many wafers broke during the slicing experiment which may be due to flat rolling. The roller should be ground to produce radiused grooves that conform to the outer radius of the diamond/nickel build-up on the wire. This would squeeze all diamonds on the cutting arc to the same elevation and not just the ones on the top surface. From this experiment it can be computed that the cutting effectiveness of bladepacks can be revived with a crushing step and that the average cutting rate for the block can be increased using this approach.

In Experiment 24 a new bladepack was "crushed" prior to slicing a $10 \mathrm{~cm}$ block. In this experiment a high yield of wafers was achieved. This data shows that it is necessary to optimize the crushing of bladepacks to extend their life and revive cutting effectiveness. 
(1)

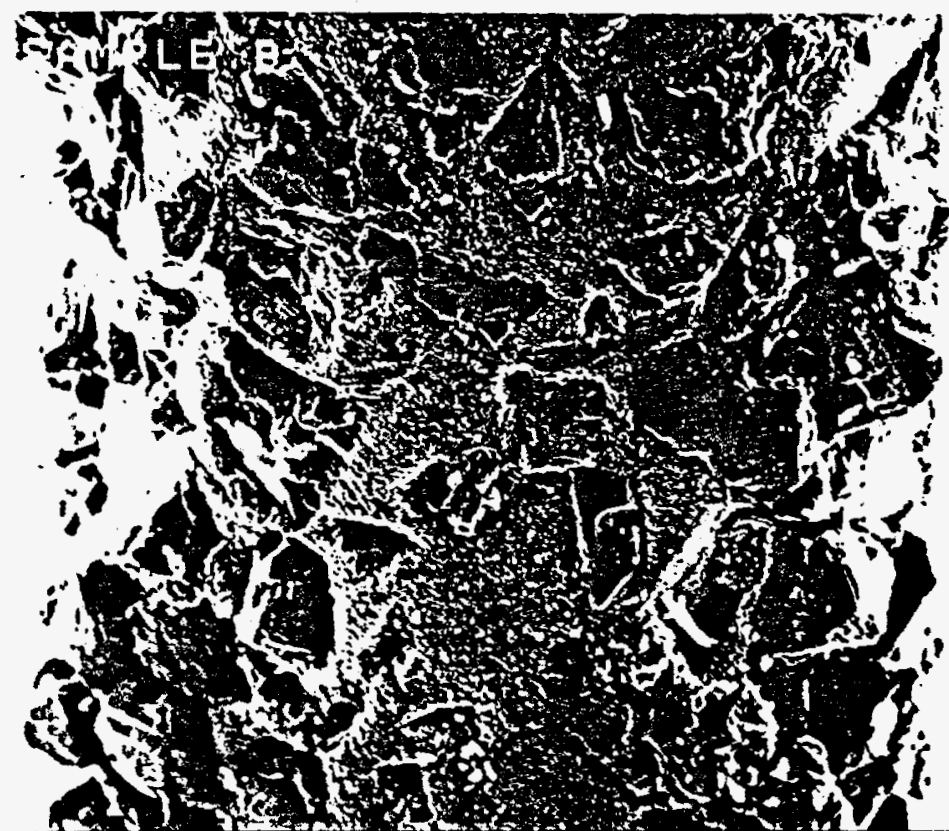

\section{$15 \mathrm{KU} 350 \mathrm{X} \quad 50 \mathrm{U} 000 \mathrm{z}$}

(ii)

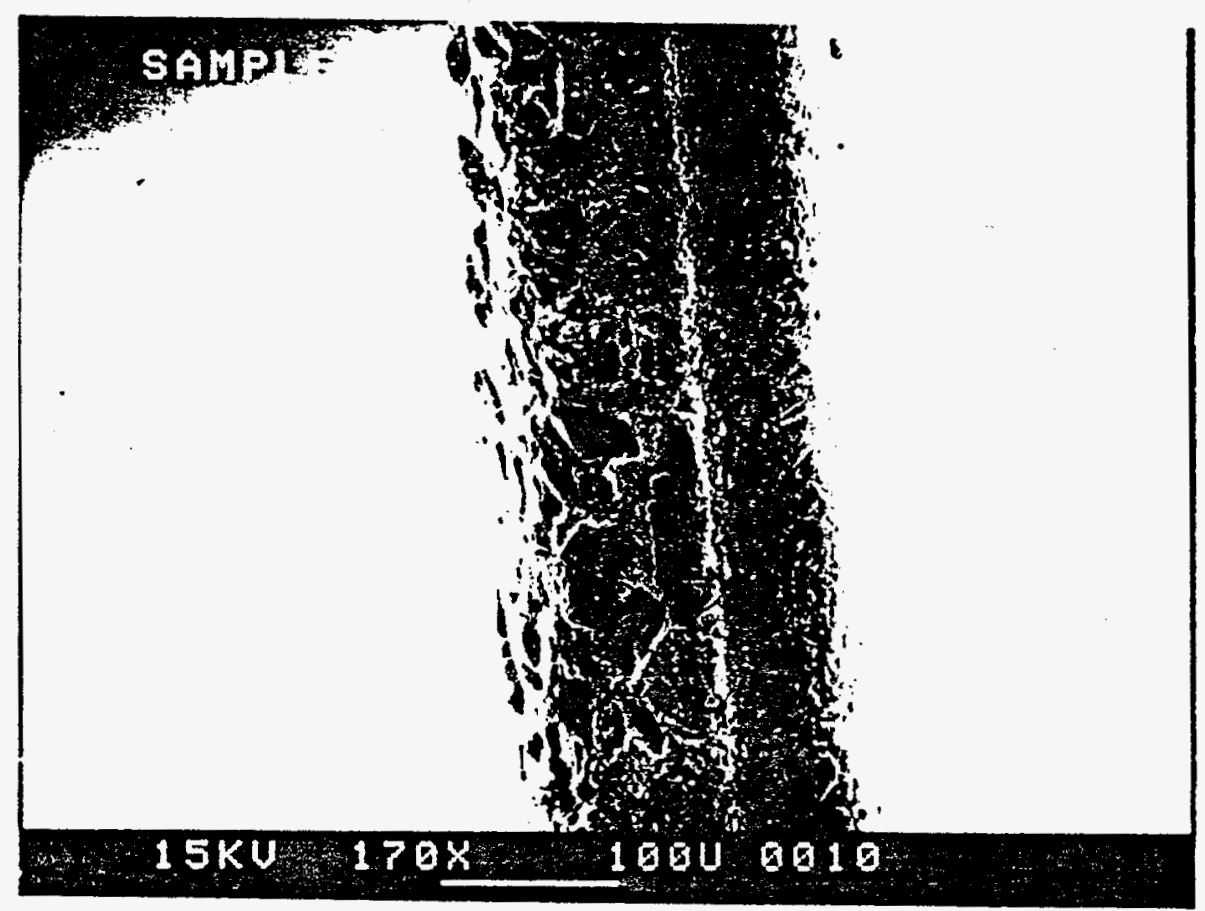

Figure 15. Two views of a wire after crushing between flat rollers showing similar protrusion of diamonds. 


\section{Increase Penetration of Diamonds into Silicon Block}

The penetration of diamonds into a workpiece, $h$, is directly proportional to the load applied, $L$, and inversely proportional to the hardness of the sample, $\mathrm{H}$, or

$$
h \propto \frac{\mathrm{L}}{\mathrm{H}}
$$

Therefore, for the same material the penetration in the sample will increase as the load is increased. The bladepack used for slicing three silicon blocks in Experiments 9,10 and 11 was used to validate the approach of slicing with higher penetration depth. A silicon block of approximately $2 \mathrm{~cm}$ width was sliced for this experiment without rocking the crystal. It was observed that the slicing rate was approximately $1.7 \mathrm{mils}$ per minute under a load of approximately $60 \mathrm{~g}$ per wire. When the load was increased to approximately $180 \mathrm{~g}$ per wire, the slicing rate increased by more than an order of magnitude (17 to 18 mils per minute). From this experiment it can be concluded that under higher load conditions, the number of diamonds involved in slicing is increased considerably by increasing the penetration of the diamonds in the workpiece.

\subsection{Degradation Mechanisms of Bladepacks}

It is important to identify the degradation mechanisms that cause the rapid degradation to diamond during FAST slicing. Stokes and Valentine ${ }^{20}$ have defined and described wear mechanisms for cubic boron nitride (CBN) abrasive. They postulate that wear of an electroplated grinding wheel may occur by the following mechanism or combinations thereof:

(i) attritious grit wear, including chemical wear

(ii) grit pull-out and bond erosion

(iii) grit fracture

(iv) wheel loading 
Examination of the nickel-diamond cutting area of the wire with both SEM (Figure 16) and optical microscope does not reveal grit pull-out, bond erosion or wheel loading. With SEM it is difficult to identify grit wear or grit fracture. The slicing experiments show that under the same test conditions, $45 \mu \mathrm{m}$ RVG friable diamond does not degrade as fast as the stronger $45 \mu \mathrm{m} \mathrm{GE} 300 \mathrm{~S}$ or $45 \mu \mathrm{m}$ friable natural or CDA diamond.

Explosively formed Dupont polycrystalline diamond is the most friable, but Experiment 19 was not meaningful. This diamond has many metal inclusions that caused large nodules to form during plating. The nickel modules may have prevented the diamond from freely contacting the work. Cubic boron nitride (CBN) is very effective for cutting and grinding hard steel, but it would not cut silicon at all, and the experiment had to be aborted immediately. Optical examination of the used wire showed massive wear flats on the CBN, as shown in Figure 17 .

SEM examination of the various types of diamond tested and shown in Figure 7 do not show any distinguishable attritious grit wear or fracture. Microfracturing of diamond is too subtle to observe with SEM.

It has been shown by SEM observation of diamond elevations, and predicted by theory, that less than 18 of the diamonds on the wire actually contact the workpiece during slicing. The forces on the diamond tips that contact the work must be high, because the blade contact length is short due to rocking to increase the pressure at the diamond tips, and there are few tips in contact within the short contact length.

Based on the results to date, it is postulated that the sharp diamond cutting tip degrades to tiny flats that act as wear surfaces. As degradation proceeds the wear flats become larger and polishing wear becomes greater and cutting due to microfracturing of silicon by sharp diamond edges becomes less 


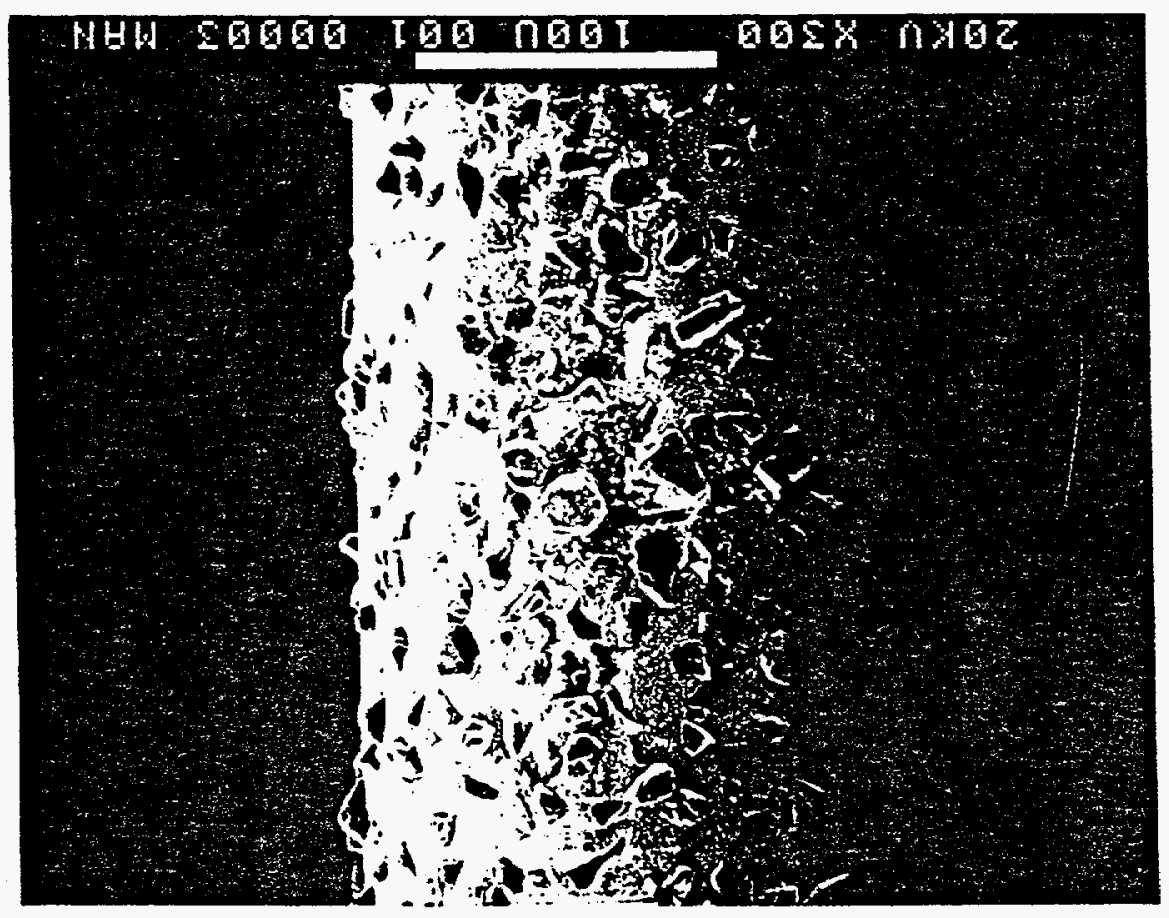


(i)

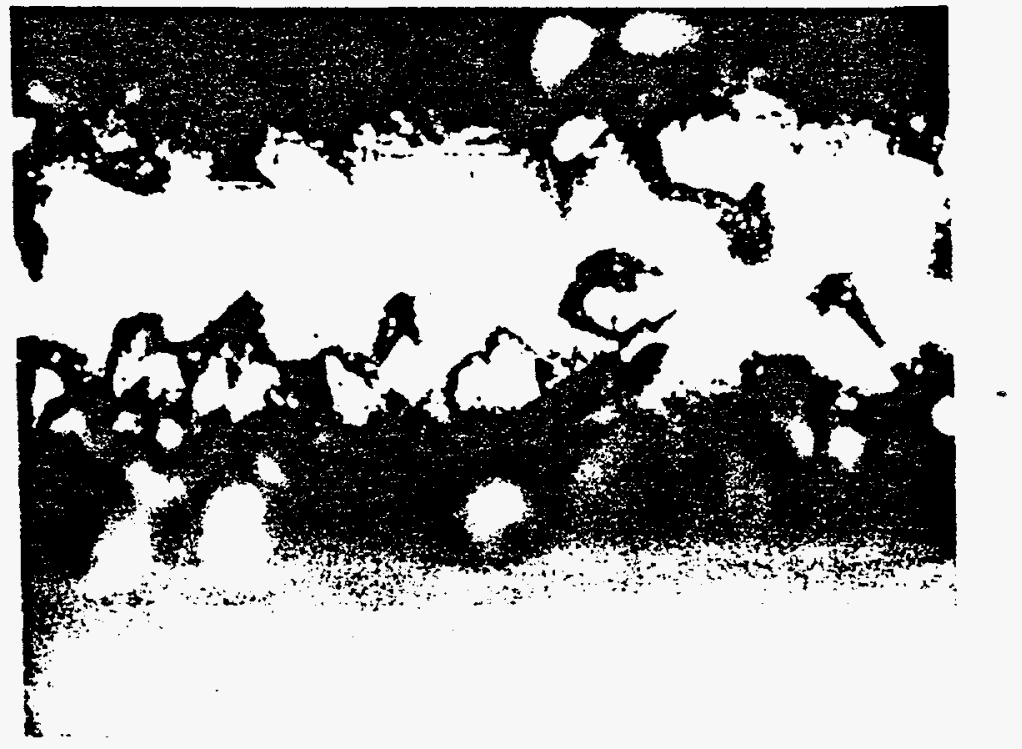

BCREN HITRIEL USEO

(ii) Higher magnification.

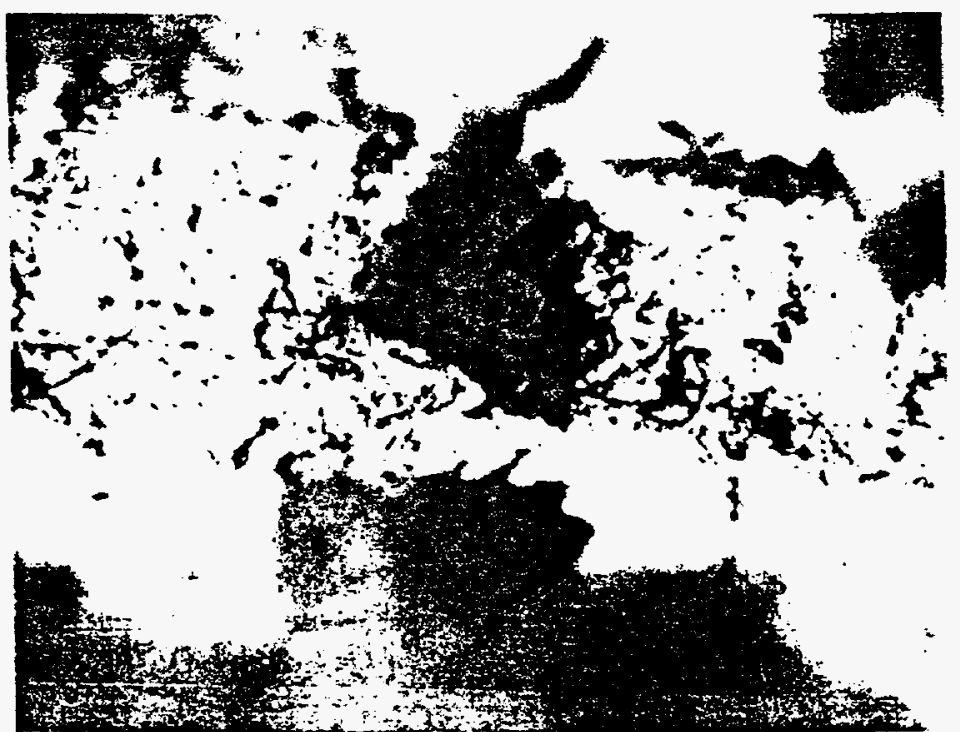

Flat on Cubjc Boron Nirit

Figure 17. Optical examination of a CBN coated wire after use showing wear flats. 
as the flats become larger. The high forces on the friable diamond may cause microfracture of the RVG diamonds to keep them sharp, or expose other diamonds. The stronger GE 300 s may not break down, but develop wear flats due to attritious wear.

For FAST the diamond tips are exposed to high loads and also to push-pull action peculiar to FAST resulting from reciprocation. The combination of high load and push-pull action may cause rotation of the diamond from a favorable to an unfavorable cutting orientation; i.e., a diamond tip that made contact with the work rotates until a more blunt surface, like a flat, contacts the work. Diamond rotation in its electroplated nickel bond has been reported for ID blades $^{21}$ where the conditions for rotation of the diamond in the nicke1 bond are not as favorable as in FAST slicing. This would suggest that the diamond should be held more firmly by using a thicker and/or harder nickel electroplating. Diamond bladepacks with the thickest electroplating resulting from longer plating time performed better than for bladepacks with thinner electroplating. Clearly the bonding of the diamond must be further investigated to see if diamond rotation to a less favorable cutting orientation is one of the degradation mechanisms for FAST. 


\subsection{Cost Analysis}

A critical element in achieving low-cost slicing is the number of cuts made with one bladepack. In addition to increasing the life of the bladepack, significant cost reductions can be achieved with FAST by slicing an increased number of wafers per linear inch of silicon. With this approach the throughput of the slicer is increased and the cost contribution from the meltstock and directional solidification of the ingot are also reduced. A comparison of the reduction of slicing costs and the effect on increasing production capacity and material utilization with FAST is shown in Table IV. This analysis has assumed that the bladepack is utilized to slice five $10 \mathrm{~cm} \times 10 \mathrm{~cm}$ silicon blocks. Even under this assumption it can be seen that the bladepack costs constitute about half the slicing costs; therefore, it is imperative that the degradation of the bladepacks during FAST slicing be minimal so that they can be utilized for slicing multiple silicon blocks. The effect of improved material utilization using FAST is shown in Table V. It can be seen that the slicing costs can be reduced by a factor of three and that the total wafer costs by a factor of two by extending the life of the bladepack and improving material utilization. 
iable IV. Material utilization, throughput and projected costs in production for FAST slicing. In this analysis the bladepack life is assumed to be 5 cuts/bladepack.

Spacing of Wires, Wires/Inch

Material Utilization

\# of wafers/inch

40

48

64

\# of wafers/cm

15.7

18.9

25.2

$100 \mathrm{~mm} \times 100 \mathrm{~mm}$ wafers produced $/ \mathrm{kg}$

68

75

100

Throughput

Cycle Time (hrs.)

16

18

20

\# of wafers/cycle

1200

1440

1920

\# of wafers/slicer/day

1800

1920

2304

\# of wafers/slicer/year

0.60

0.64

0.77

(333 days/yr.), million

\section{Projected Costs}

Direct Labor (5 slicers/operator, $\$ 12 / \mathrm{hr}.), \$ /$ Wafer

0.0320

0.0300

0.0250

Indirect Labor

$\underline{0.0320}$

$\underline{0.0300}$

$\underline{0.0250}$

Total Labor, \$/Wafer

0.0640

0.0600

0.0500

\section{Equipment Costs}

$(\$ 250,000$ slicer, 5 yr. 11fe $), \$ /$ Wafer

0.0834

0.0782

0.0652

Expendable Materials

Other - (\$25/run), \$/Wafer

0.0208

0.0174

0.0130

Bladepack - $(\$ 500,550,605$ each,

5 cuts/bladepack), \$/Wafer

$\underline{0.1667 \quad \underline{0.1526} \quad \underline{0.1260}}$

Total Costs, $\$ /$ wafer

0.3349

0.3082

0.2542 
Table V. Projected costs of FAST slicing, \$/wafer, showing sensitivity of bladepack life and material utilization on silicon ingot, slicing and total wafer costs for $100 \mathrm{~mm} \times 100 \mathrm{~mm}$ wafer production. Silicon bar costs are assumed to be $\$ 70 / \mathrm{kg}$ and other parameters are the same as Table IV.

\begin{tabular}{|c|c|c|c|c|}
\hline \multirow[t]{2}{*}{ \# of Wafers/Inch } & \multicolumn{3}{|c|}{ \# of Cuts/Bladepack } & \\
\hline & 2 & $\underline{5}$ & 10 & \\
\hline 40 & $\begin{array}{l}1.0357 \\
0.6349 \\
1.6706\end{array}$ & $\begin{array}{l}1.0357 \\
0.3349 \\
1.3706\end{array}$ & $\begin{array}{l}1.0357 \\
0.2515 \\
1.2872\end{array}$ & $\begin{array}{l}\text {-Silicon Bar } \\
\text {-Slicing } \\
\text {-Wafer Costs }\end{array}$ \\
\hline 48 & $\begin{array}{l}0.8631 \\
0.5375 \\
1.4006\end{array}$ & $\begin{array}{l}0.8631 \\
0.3082 \\
1.1713\end{array}$ & $\begin{array}{l}0.8631 \\
0.2320 \\
1.0951\end{array}$ & $\begin{array}{l}\text {-Silicon Bar } \\
\text {-Slicing } \\
\text {-Wafer Costs }\end{array}$ \\
\hline 64 & $\begin{array}{l}0.6473 \\
0.4433 \\
1.0906\end{array}$ & $\begin{array}{l}0.6473 \\
0.2542 \\
0.9015\end{array}$ & $\begin{array}{l}0.6473 \\
0.1912 \\
0.8385\end{array}$ & $\begin{array}{l}\text {-Silicon Bar } \\
\text {-Slicing } \\
\text { - Wafer Costs }\end{array}$ \\
\hline
\end{tabular}




\subsection{Results and Discussions}

The photovoltaic industry has been growing at an annual rate projected at 20 to 30 percent. To broaden photovoltaic applications it is necessary to lower the cost of modules without sacrificing high performance. The photovoltaic industry recognizes that only crystalline silicon can meet the requirements of photovoltaic modules for near future applications. To lower costs the highpower density must be combined with efficient high-throughput in production.

Silicon wafer cost represents more than half total module cost; reduction of silicon wafer cost is therefore critical for lowering the costs of photovoltaic energy. During the last decade crystalline silicon ingot growth technologies have shown considerable progress in producing high-efficiency material at low cost; however, slicing of these ingots is still an obstacle since it represents nearly 50 percent of the wafer cost, and the expendable blade cost represents about one half the slicing cost. It is desirable to develop a high production slicing technique to slice thin wafers with low kerf at low cost. If these kerf and wafer objectives are achieved, the contribution of silicon meltstock and ingot growth costs to the wafer costs are lessened because less silicon material is required. The key to reducing the slicing cost is to reduce the expendable costs.

Among the slicing techniques available to the industry, the ID technique has been optimized, and significant further cost reductions are not anticipated. Improvements have been made in NWS technique; however, expendable materials costs control the cost of slicing. Moreover, the disposal of silicon, silicon carbide and oil mixture presents environmental problems.

A third approach, FAST, is currently at a prototype production stage. In FAST the expendable materials costs are reduced by using a diamond-plated wirepack, while the advantages of NWS are retained. Once FAST slicing was 
demonstrated in the laboratory and it was shown that high quality thin wafers could be produced with low kerf, a major development program was undertaken in 1989. The emphasis in this development program was to design and fabricate a prototype FAST slicer with increased throughput and potentially to lower cost in production. A double-headed prototype FAST slicer with a capacity to slice 30 inches of silicon bar per run was designed and built. Effective slicing at 40 wafers per inch has demonstrated that 1,200 wafers can be produced per run. Thinner wafers can be sliced by changing the spacing of the wires in the bladepack.

Simultaneously with the slicing machine development, emphasis was placed on developing of effective wirepacks for FAST slicing. Effective electroforming techniques have been developed which minimize kerf and reduce costs. It has been observed that cutting effectiveness of bladepacks degrades with time. Developments in plating bladepacks have shown that diamond pullout during FAST slicing is no longer a problem.

The present program on wire blade development was initiated to understand the degradation mechanism of the wirepacks. Experimental evidence showed that a very small number of diamonds on the wire contact the silicon workpiece during FAST slicing; therefore, the effective feed forces on each diamond tip is high. Under these conditions the diamond tip may become blunt by forming a "wear flat" or it may be moved in its nickel setting to a less favorable cutting orientation during the reciprocation action. Since the effective pressure on the diamond tip decreases as the flat increases in size, the diamond cannot be removed from the wire to allow diamonds at lower elevations to contact the workpiece; effective slicing cannot be restored. Once a silicon block is sliced, the diamonds at highest elevations can be removed by dressing so that sharp diamonds at lower elevations contact the workpiece. Therefore, cutting effectiveness of 
a wirepack can be restored after dressing and prior to slicing a new silicon block. Using this approach the wirepack life has been increased from slicing a silicon block to slicing of three silicon blocks.

It has not been established that the degradation mechanism for wirepacks is due to a very small number of diamonds contacting the workpiece during FAST slicing. A theoretical analysis is being carried out to confirm the experimental observation of the degradation mechanism. Once the degradation mechanism was identified, an attempt was made to crush roll the wirepack so that the number of diamonds at the same elevation were increased and more diamonds contacted the workpiece during slicing. The results were positive. An attempt was also made to increase the number of diamonds involved in FAST slicing by slicing at higher feed forces. Slicing rates were increased by an order of magnitude with a three-fold increase in the feed force.

At the present time an effective high-throughput FAST slicer has been installed. The basic development to produce electroformed wirepacks with a high concentration of diamonds plated only in the cutting edge of the wires has been completed. Effective slicing has been demonstrated with surface damage in FAST sliced wafers limited to 3 to $5 \mathrm{~mm}$. At the present time the expendable materials costs for FAST slicing are high because the cutting effectiveness of the wirepacks is degraded during slicing. The mechanism for loss in cutting effectiveness has not been identified. It has been shown that the cutting effectiveness can be revived after FAST slicing by a dressing operation. During this dressing step very few diamonds are removed so that the diamond concentration for slicing the subsequent silicon block is still high. The theoretical calculations complement the experimental model for the degradation mechanism for the wirepacks. 
A number of other wire configurations and techniques have now been identified which could aid FAST slicing so that a wirepack can slice up to ten $10 \mathrm{~cm}$ square silicon blocks. Once this is established in practice, the wire spacing can be changed to improve the material utilization. Further wafer economies can be achieved by reducing the silicon material utilized in each wafer.

Therefore, FAST has the potential of achieving major cost reductions for silicon wafers and make photovoltaics a viable energy technology. 


\subsection{References}

1. Crystal Systems, Inc., DOE/JPL Contract No. 954373, Silicon Ingot Casting Heat Exchanger Method, Multi-Wire Slicing - Fixed Abrasive Slicing Technique, Nov. 1975 - June 1981.

2. F. Schmid, U. S. Patent No. 4,178,670, Dec. 18, 1979.

3. F. Schmid, M. B. Smith and C. P. Khattak, U. S. Patent No. 4,384,564, May 24, 1983.

4. F. Schmid, M. B. Smith and C. P. Khattak, U. S. Patent No. 4,646,710, March 3, 1987.

5. F. Schmid, M. B. Smith and C. P. Khattak, U. S. Patent No. 4,727,852, March 1, 1988.

6. F. Schmid, C. P. Khattak, M. B. Smith and L. D. Lynch, Proc. 4th E. C.

1 Photovoltaic Solar Energy Conf., D. Reidel Pub1. Co. (1982) p. 980.

7. C. P. Khattak and F. Schmid, Proc. 2nd E. C. Photovoltaic Solar Energy Conf., D. Reidel Publ. Co. (1979) p. 106.

8. C. P. Khattak, F. Schmid and M. B. Smith, Proc. of the Low-Cost Solar Array Wafering Workshop, Phoenix, AZ, June 1981, DOE/JPL-1012-66 (1982) p. 111.

9. F. Schmid and C. P. Khattak, HEM - Ingot Casting; FAST - Multi-Wire S1icing, Phase II Final Report, DOE/JPL 954373-79/9, June 1979.

10. F. Schmid and C. P. Khattak, HEM - Ingot Casting; FAST - Multi-Wire Slicing, Phase I Final Report, DOE/JPL 954373-77/4, Dec. 1977.

11. F. Schmid, M. B. Smith and C. P. Khattak, Proc. of the Low-Cost Solar Array Wafering Workshop, Phoenix, AZ, June 1981, DOE/JPL-1012-66 (1982) p. 233.

12. R. P! Lindsay, Principles of Grinding, in R. I. King and R. S. Hahn, eds., Handbook of Modern Grinding Technology (Chapman and Hall, London, 1986).

13. K. H. Zum Gahr, Microstructure and Wear of Materials (Elsevier, Amsterdam, 1987).

14. A. A. Torrance, Wear 118, 217 (1987).

15. A. A. Torrance, Wear 139, 383 (1990).

16. T. C. Buttery and J. F. Archard, Proc. Instn. Mech. Engrs. 185, 537 (1971).

17. J. L. Metzger and A. A. Torrance, Industrial Diamond Review 50(6), 296 (1990).

18. A. A. Torrance and J. L. Metzger, Industrial Diamond Review, 1991, p. 14.

19. J. F. Archard, Wear 2, $438(1958 / 59)$.

20. R. J. Stokes and T. J. Valentine, Industrial Diamond Review, 1984, p. 34.

21. Boujikian, Proc. of the Low-Cost Solar Array Wafering Workshop, Phoenix, AZ, June 1981, DOE/JPL-1012-66 (1982) p. 71. 


\begin{tabular}{|c|c|c|c|}
\hline $\begin{array}{l}\text { Document Control } \\
\text { Page }\end{array}$ & $\begin{array}{l}\text { 1. SERI Report No. } \\
\text { NREL/TP-214-4485 }\end{array}$ & $\begin{array}{l}\text { 2. NTIS Accession No. } \\
\text { DE92001165 }\end{array}$ & 3. Recipient's Accession No. \\
\hline \multirow{2}{*}{\multicolumn{3}{|c|}{$\begin{array}{l}\text { 4. Title and Subtitle } \\
\text { Development of a Fixed Abrasive Slicing Technique (FAST) for Reducing the } \\
\text { Cost of Photovoltaic Wafers }\end{array}$}} & $\begin{array}{l}\text { 5. Publication Date } \\
\text { December } 1991\end{array}$ \\
\hline & & & 6. \\
\hline $\begin{array}{l}\text { 7. Author(s) } \\
\text { F. Schmid }\end{array}$ & & & 8. Performing Organization Rept. No. \\
\hline \multirow{2}{*}{\multicolumn{3}{|c|}{$\begin{array}{l}\text { 9. Performing Organization Name and Address } \\
\text { Crystal Systems Inc. } \\
27 \text { Congress St. } \\
\text { Salem, Massachusetts } 01970\end{array}$}} & $\begin{array}{l}\text { 10. Project/TaskWork Unit No. } \\
\text { PV150101 }\end{array}$ \\
\hline & & & $\begin{array}{l}\text { 11. Contract (C) or Grant (G) No. } \\
\text { (C) XC-1-10057-17 } \\
\text { (G) }\end{array}$ \\
\hline \multirow{2}{*}{\multicolumn{3}{|c|}{$\begin{array}{l}\text { 12. Sponsoring Organization Name and Address } \\
\text { National Renewable Energy Laboratory } \\
1617 \text { Cole Blvd. } \\
\text { Golden, CO } 80401-3393\end{array}$}} & $\begin{array}{l}\text { 13. Type of Report \& Period Covered } \\
\text { Technical Report }\end{array}$ \\
\hline & & & 14. \\
\hline \multicolumn{4}{|c|}{$\begin{array}{l}\text { 15. Supplementary Notes } \\
\text { NREL technical monitor: R. Mitchell }\end{array}$} \\
\hline \multicolumn{4}{|c|}{$\begin{array}{l}\text { 16. Abstract (Limit: } 200 \text { words) } \\
\text { This report examines a wafer slicing technique } \\
\text { wafers. This fixed, abrasive slicing technique (F } \\
\text { coolant. FAST is in the prototype production sta } \\
\text { multiwire slurry technique. The cost analysis re } \\
\text { slicing more wafers per linear inch. Researchers }\end{array}$} \\
\hline \multicolumn{4}{|c|}{$\begin{array}{l}\text { 17. Document Analysis } \\
\text { a. Descriptors } \\
\text { fixed, abrasive slicing ; photovoltaic wafers } \\
\text { b. Identlfiers/Open-Ended Terms }\end{array}$} \\
\hline \multicolumn{4}{|l|}{$\begin{array}{l}\text { c. UC Categories } \\
274\end{array}$} \\
\hline \multirow{2}{*}{\multicolumn{2}{|c|}{$\begin{array}{l}\text { 18. Availability Statement } \\
\text { National Technical Information Service } \\
\text { U.S. Department of Commerce } \\
\text { 5285 Port Royal Road } \\
\text { Springfield, VA } 22161\end{array}$}} & & $\begin{array}{l}\text { 19. No. of Pages } \\
64 \\
\end{array}$ \\
\hline & & & $\begin{array}{l}\text { 20. Price } \\
\text { A04 }\end{array}$ \\
\hline
\end{tabular}

Form No. 0069E (6-30-87) 